\title{
ON INTERACTION BETWEEN FALLING BODIES AND THE SURROUNDING FLUID
}

\author{
FRANK T. SMITH AND ANDREW S. ELLIS
}

\begin{abstract}
Interactions between a finite number of bodies and the surrounding fluid, in a channel for instance, are investigated theoretically. In the planar model here the bodies or modelled grains are thin solid bodies free to move in a nearly parallel formation within a quasi-inviscid fluid. The investigation involves numerical and analytical studies and comparisons. The three main features that appear are a linear instability about a state of uniform motion, a clashing of the bodies (or of a body with a side wall) within a finite scaled time when nonlinear interaction takes effect, and a continuum-limit description of the body-fluid interaction holding for the case of many bodies.
\end{abstract}

$\S 1$. Introduction. The study of interactions between moving solid bodies and the surrounding fluid has many natural, industrial and biomedical applications. These and background motivations are considered in $\S 1.1$. Previous studies are discussed in $\S 1.2$, and $\S 1.3$ focuses on the present work.

1.1. Applications and motivation. A great many applications arise across nature such as with falling leaves and moving seeds and coffee grains (e.g. [4, $7,27,52]$ ), not to forget the motion of frozen ice particles and hailstones as well as sedimentation and fluidization phenomena. Applications also arise in sporting contexts such as running and cycling groups and to some extent in longdistance swimming competitions. The behaviours of various swarms similarly have an interactive fluid-dynamical element to them, while a communication to the authors has pointed out the collisions of ships due to rushing water and suction between them, as occurred for example during the UK-Iceland cod war and the similar danger of suction for barges in the Suez Canal.

Three industrial applications are concerned with the falling of lumps of ice into an engine intake in an aerodynamic safety context, the travel of windblown particles of ice along a wing surface again in the aerodynamic safety context, and the falling of rice grains down a chute in a food-sorting context. In addition various disintegration, deposition, oil-well and sequestration modelling applications exist for interactions between solid bodies and fluids.

There are also many biomedical applications in principle, for example to travel of solids within vessels of major networks in the human body. Specific applications are to transport of blood clots, embolization procedures in stroke 
treatment (transportation of glue), drug delivery to tumours via a capillary network, the passage of cells through vessels of lateral dimension comparable with the effective cell diameter, and deposition of tiny particles in branching systems $[3,5,6,9,10,12,18,21,24,37,42,47-49,53]$. One fundamental question is how far and where small objects will travel when transported, which is a global network issue as well as depending on the shapes of the objects and of the local vessels.

The solid-fluid interactions of general interest are with or without side walls being present, although in the food-sorting application which we keep tentatively in mind there is interest in the shaping of the chute side walls. In the latter application the grains travel as a monolayer down an inclined chute (which has a free-surface top), fall from the bottom of the chute and then pass an optical system that can detect defective grains. A powerful jet of air is fired to eject a defective grain. Since the grains falling off the chute typically are not uniformly distributed but clustered and inhomogeneous, the air jet removes other grains surrounding the target grain which may not themselves be defective. An industrial goal is to increase uniformity of "product feed" to reduce the ejection difficulty whilst maintaining a high throughput of grains. The ideal situation is for an evenly spaced and uniformly ordered array of grains to fall down and off the chute such that each grain is aligned with an ejector. This points to a study of air effects on arrays of grains and the role of the chute wall.

Numerical estimates concerning food sorting are of interest here. The chutebased global Reynolds number of the flow is $\operatorname{Re}=L^{*} V^{*} / \nu^{*}$ where $L^{*}(\approx 1 \mathrm{~m})$ is the chute length, $V^{*} \approx 4.5 \mathrm{~m} \mathrm{~s}^{-1}$ is the grains' terminal velocity and $v^{*} \approx$ $1.5 \times 10^{-5} \mathrm{~m}^{2} \mathrm{~s}^{-1}$ is the kinematic viscosity of air. Thus $\operatorname{Re} \approx 3 \times 10^{5}$ and therefore the dimensional boundary layer thickness $\delta^{*}=O\left(\operatorname{Re}^{-1 / 2} L^{*}\right) \approx 2 \mathrm{~mm}$; hence the global boundary layer thickness is of the order of the grain size (see below), and so we might expect inviscid air effects to be significant toward the bottom of the chute. On the other hand the grain-based local Reynolds number is given by $l^{*} V^{*} / \nu^{*}$ with $l^{*}$ now being the length of a rice grain, which is typically of the order of $5 \mathrm{~mm}$. Thus locally $\mathrm{Re} \approx 1.5 \times 10^{3}$. The local dimensional boundary layer thickness is then approximately $0.1 \mathrm{~mm}$, which is nominally small relative to the grain size. Overall, then, a nominal inviscid approximation seems reasonable.

Aspects of the above applications motivate, however remotely, the present study into body-fluid interactions. The present paper will refer to the immersed bodies as "grains", considering them as long or thin bodies. These merit particular attention because in principle they overlap and align readily compared with spherical grains, for example, and so are potentially suitable for sorting purposes among others.

1.2. Previous studies. Concerning existing granular flow theories, usually problems in granular mechanics deal with phenomena such as avalanches, lahars (landslides), pharmaceutical processes, powders in rotating drums and chute flows $[19,35,40]$. There are some well-developed theories of chute flows $[1,14,25,26,33]$; typically even for sparser rapid granular flows enduring 
particle contacts are significant and the flows can be many particles deep. A standard approach here is to postulate interesting so-called "constitutive relations" that are crucial to the granular flow $[\mathbf{1}, \mathbf{1 1}, \mathbf{2 5}, \mathbf{2 6}, \mathbf{2 9}, \mathbf{4 0}]$. These studies often address the particle forces alone, neglecting the multiphase nature of granular materials, especially for rapid flows [11]. The constitutive relations typically focus on the shear forces and stress tensors obtained from the particleparticle collisions, and the notion of granular temperature is often introduced as an analogue to kinetic theories of ideal gases.

On the other hand, suspension flow studies tend to be concerned with sparser grain flows where the interstitial fluid is important for the grain dynamics $[13, \mathbf{2 2}, \mathbf{3 0}, \mathbf{5 5}, \mathbf{5 6}]$, such as in the study of aeolian or fluvial transport [54]. These studies address interesting issues such as entrainment, which are potentially relevant to some body-fluid interactions of present concern.

Other investigations include relevant works by Guazzelli [20], for example on oscillations in sedimentation of spheres and fibres, bubbles in fluidized beds and migration of particles, and $[34,38,57]$ on bubble formations and clusters. The nonlinear multi-body interactions addressed by $[\mathbf{8}, \mathbf{3 9}, \mathbf{4 3}, 44]$ for in-series wakes and by $[37,45,46]$ for internal branching motions are also relevant even though they assume fixed bodies in steady flow.

1.3. The present study. The rapid monolayers in sorting and related applications, for example, appear to be atypical for a granular flow, and enduring contacts are not thought to be as significant here; frequent binary impacts are more typical. The issues involved seem to lie between or outside the arenas of existing granular flows and suspension flows. The current focus is on substantial interactions in which the fluid flow is at relatively high rates producing rather a flow multi-structure. The grains respond nonlinearly by means of their own induced motion which in turn affects the fluid flow nonlinearly. In terms of grains falling down and off a chute, for example, we concentrate on the grainfluid interactions occurring at the lower end of the chute where the fluid response is effectively inviscid due to the increased velocity of the grains. The upper part of the chute where viscous or viscous-inviscid behaviour is more appropriate is considered in [15], while a continuum model for the bulk properties of the grain motion without air effects is addressed in [16].

For the comparatively fast motion of grains when they are in a nearly parallel formation within the surrounding fluid, a two-dimensional unsteady flow approximation is taken here as a starting point, from which the main findings should extend to three dimensions except for swirl and similar effects. The primary aim is to investigate a model for nonlinear interactions between one or more falling grains of uniform density contained within side walls. The investigation aims to examine linear stability properties concerning the ideal of a perfectly parallel motion of the grains and then nonlinear behaviour, the possibility of contacts or clashes between the grains, the issue of a rational continuum limit for the case of many grains, and even to gain some insight into bouncing of the grains after clashing occurs. This, to repeat, is all within the confines of a two-dimensional theory which may neglect significant threedimensional effects such as swirling of the fluid as well as the grains. 
Section 2 describes the grain-flow interaction model and solution structure which leads to a nonlinear system of difference, longitudinal-differential and integral equations for the motion of one or a finite number of grains in surrounding fluid. Section 3 examines the linear and nonlinear evolution properties for various cases of configurations. Most cases are found to yield a clash of one or more grains at the leading edge of the configuration within a finite scaled time, in contrast with previous interesting work on single grains or other bodies in quite different settings $[\mathbf{3 1}, \mathbf{3 2}, \mathbf{3 6}, \mathbf{4 1}, \mathbf{5 0}, \mathbf{5 9}]$, but possibly tying in with earlier work $[\mathbf{2}, \mathbf{1 7}, \mathbf{2 3}, \mathbf{5 8}]$. The typical present clash is examined in detail in $\S 4$. The continuum limit is then explored in $\S 5$, leading to lateraldifferential and integral dependence coming into play. Further comments, which include reconsideration of the sorting application and three-dimensionality as well as removal of the side wall effects, are made in $\S 6$.

§2. Fluid-grain interaction and governing equations. The configuration of concern here has grains falling through fluid down a vertical chute as drawn in Figure 1. The model interactions between grains and fluid which we study assume that the fluid is in effect incompressible and inviscid and that, as a starting point, the entire motion takes place in a two-dimensional plane marked out by Cartesian coordinates $x^{*}, y^{*}$ as shown, with an asterisk signifying a dimensional quantity. Thus the grains themselves are taken as two-dimensional and in addition it is assumed, again as a starting point, that they are nearly vertical in a sense to be defined. The focus here is on thin straight grains, effectively flat plates as in Figure 1, although the same flow-solid interaction structure applies to cases with thickness and camber. A single thin straight grain with side walls is shown in Figure 1(a). This is extended to multiple grains in Figures 1(b) and (c); in Figure 1(c) their leading edges are aligned with each other, as are their trailing edges. The grains form an unknown row-like pattern and they are finite in number. (Concerning the food-sorting context, there are no back wall effects included as the presence of the chute manifests itself only as side walls as for a channel in the $x^{*}-y^{*}$ plane.) There are still at least two significant length scales. The representative vertical or longitudinal extent of the grains from leading to trailing edge is $L_{1}^{*}$ and the horizontal or lateral distance in $y^{*}$ is $L_{2}^{*}$. The typical speed of descent over the time scales of interest is written as $U^{*}$ and is taken as given, having been determined by gravity, air resistance and other forces during the descent. Velocity components are $u^{*}, v^{*}$, pressure is $p^{*}, t^{*}$ denotes the time and $\rho^{*}$ is the fluid density.

The interaction structure below holds for all the configurations in Figure 1. In Figure 1(b) the second group (the upper group, which has four grains) and any subsequent groups of grains have negligible influence on the first group (of five grains) however, and interaction for the first group involves an extension of that for the aligned configuration in Figure 1(c) anyway. So we concentrate below on the cases of Figure 1(a) and (c). Further, the restriction to two spatial dimensions at this stage has been explained already, although some similar features are to be expected in three dimensions with small gaps present. 
(a)

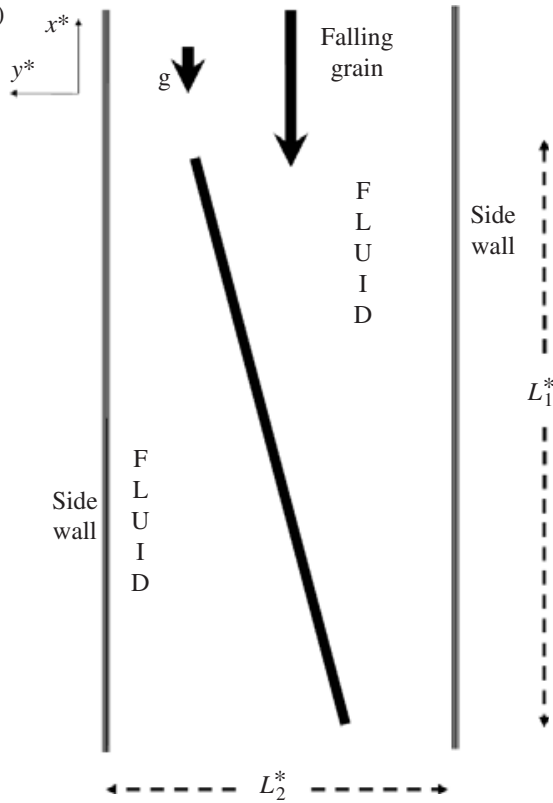

(b)

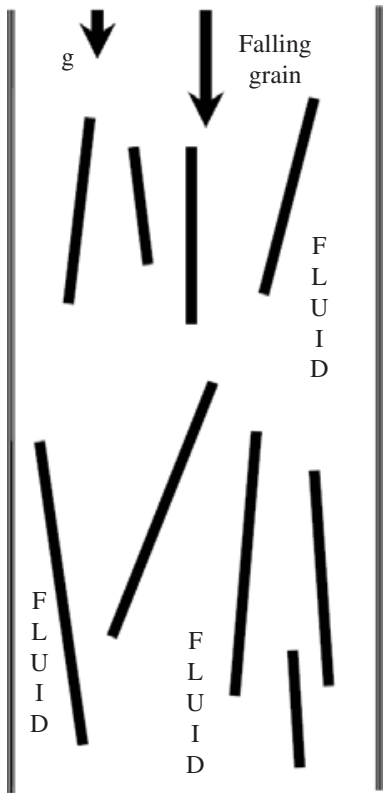

(c)

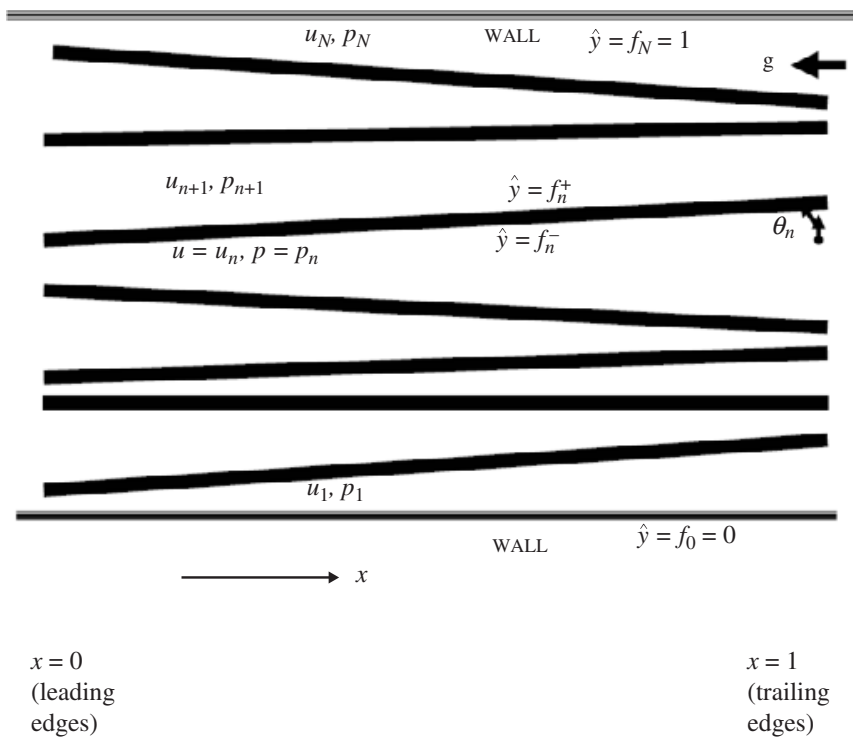

Figure 1: Sketches (not to scale) of (a) a single thin straight grain falling through fluid, (b) more grains falling, with a leading group of five and a second group of four, and (c) many grains, with aligned leading and trailing edges, showing the main notation and interaction features involved, where for convenience (c) has been rotated through 90 degrees relative to (a) and (b). The paper concentrates mostly on the configurations in (a) and (c). 
Non-dimensional quantities are to be used for convenience in a frame moving down vertically with the grains at speed $U^{*}$, such that

$$
\begin{gathered}
x^{*}-x_{0}^{*}=L_{1}^{*} x, \quad y^{*}=L_{1}^{*} y, \quad\left(u^{*} v^{*}\right)=U^{*}(u, v), \\
p^{*}=\rho^{*} U^{* 2} p, \quad t^{*}=L_{1}^{*} t / U^{*} .
\end{gathered}
$$

Here we choose $L_{1}^{*}$ as the main dimensional length scale for convenience, which makes the typical range of $x$ be unity and that of $y$ be $\beta$, where $\beta \equiv$ $L_{2}^{*} / L_{1}^{*}$. Also $u, v$ are the fluid or grain velocity components in terms of $x, y$ respectively, $p$ is the dynamic pressure to within a constant and $t$ denotes time in non-dimensional form with an origin shift. The location $x_{0}^{*}$ allows for the shift in the longitudinal origin associated with the original grain descent relative to the side walls, and this is taken to be moving at effectively constant speed during the present grain-fluid interactions. The governing equations in full for the fluid are the continuity and Navier-Stokes equations and for the grains are those of rigid body motion. The fluid and the grains interact by virtue of the unknown movements of the individual grains when subjected to fluid dynamic forces and the equally unknown flow of the fluid affected by the moving boundaries.

Our interest in most of this paper is in the cases where the characteristic length ratio $\beta=L_{2}^{*} / L_{1}^{*}$, which is also a measure for the typical angle of the fluid flow vectors within the fluid gaps and for the inclinations of the grains from the vertical, is small; $\$ 6$ considers extending this. The finitely many gaps have thicknesses of order $\beta$ in $y$ and so the flow solution expands in the fashion

$$
(u, v, p)=\left(u_{n}, \beta \hat{v}_{n}, p_{n}\right)+\cdots \quad \text { with } x, t \text { of } O(1) \text { and } y=\beta \hat{y},
$$

in the majority of the flow field. The typical size of $y$ represents the distance across the chute as well as the fluid gap thicknesses, while that of $v$ in (2.2) is inferred from the continuity equation. Here $u_{n}, \hat{v}_{n}, p_{n}, \hat{y}$ are of order unity, and $n$ runs from 1 to $N$ in the successive $N$ gaps for $N-1$ grains in a row between the side walls $\hat{y}=0$ and $\hat{y}=1$. The integer $N \geq 2$. The fluid flow equations in each gap are therefore those of thin inviscid layers,

$$
\begin{gathered}
\partial u_{n} / \partial x+\partial \hat{v}_{n} / \partial \hat{y}=0, \\
\partial u_{n} / \partial t+u_{n} \partial u_{n} / \partial x+\hat{v}_{n} \partial u_{n} / \partial \hat{y}=-\partial p_{n} / \partial x, \\
0=-\partial p_{n} / \partial \hat{y},
\end{gathered}
$$

from the balances of continuity, longitudinal momentum and lateral momentum respectively. These hold provided not only that $\beta$ is small but also $\beta^{2} \operatorname{Re}$ is large, as we shall suppose, where Re is the characteristic longitudinal Reynolds number $U^{*} L_{1}^{*} / \nu^{*}$ and $v^{*}$ is the kinematic viscosity of the fluid. The negligible inertial impact in (2.3c) implies that $p_{n}$ is an unknown function of $x, t$ only. For each grain and gap $x$ lies between zero and unity longitudinally, while laterally the total extent in $\hat{y}$ is between zero at one wall and unity at the other. The oncoming fluid motion effectively has $u_{n}=1, \hat{v}_{n}=0, p_{n}=0$ due to the frame of reference. We note also that gravity as well as viscous and wall-contact effects 
are neglected during the current interactions, with gravity having served to fix the typical descent (terminal) speed as mentioned previously. The boundary conditions in the lateral sense are the kinematic ones

$$
\hat{v}_{n}=\partial f_{m} / \partial t+u_{n} \partial f_{m} / \partial x \text { on } \hat{y}=f_{m}(x, t) \quad \text { with } m=n, n-1
$$

for each solid grain boundary given by the unknown position $f_{n}(x, t), n=1$ to $N-1$, and

$$
\hat{v}_{1}=0 \text { on } \hat{y}=0, \quad \hat{v}_{N}=0 \text { on } \hat{y}=1,
$$

for tangential flow at the straight solid side walls. (See also the comments about flow separation below.) Here (2.3d) is for cases where the grains are thin compared with the gap widths, our main cases of concern, although significant grain thickness can be incorporated by allowing different $f_{n}$ (say, $f_{n}{ }^{ \pm}$) variations on either side of each grain.

At the trailing edges where $x$ is unity, Kutta conditions apply as the individual gap flows enter into the common wake, requiring the pressures across all the gaps to be equal there,

$$
p_{1}=p_{2}=\cdots=p_{N} \quad \text { at } x=1 .
$$

In contrast the velocities $u_{n}$ at the trailing edges are unequal generally, admitting vortex sheets into the common wake.

A further significant feature which is associated with upstream influence in the whole system is that streamwise jumps (Euler jumps, as in [8]), in pressure must be allowed at the leading-edge station $(x=0)$. The reason stems from the hyperbolic nature of the gap flows in (2.3a)-(2.3c), which indicates zero upstream influence in general and so a possible contradiction with the equipressure requirement $(2.3 \mathrm{f})$ at each trailing edge as the different gap flows usually produce different pressures at the trailing edges if they begin with identical leading-edge pressures. The resolution is provided by a flow solution discontinuity which can occur in a self-consistent manner only in the vicinity of the leading edges, where all the upstream influence is focused in a sense. It follows that in general the incident pressure of zero just ahead of the $n$th leading edge is different from the two distinct values $p_{n}(0+, t), p_{n+1}(0+, t)\left(\pi_{n}, \pi_{n+1}\right.$ respectively, say) holding on either side of the $n$th grain just downstream. Instead the scaled Bernoulli quantity $p+\frac{1}{2} u^{2}$ and the scaled vorticity are conserved across the leading-edge station and give rise to the conditions that $p+\frac{1}{2} u^{2}=\frac{1}{2}$ and $\partial u / \partial y$ is zero at the onset of each gap flow, in view of the incident uniform stream and pressures.

The streamwise length scale involved in the comparatively short region necessary to smooth out the leading-edge jumps is of order $\beta$, the same as the lateral scale of the gaps, and the sizes of the velocity components and pressure are clearly given by $u \sim v \sim 1, p \sim 1$ respectively. Hence the leading-edge region is controlled by quasi-steady planar Euler dynamics spanning the entire chute from wall to wall, with the thin grains appearing as flat plates aligned with the incident uniform stream, and that scenario leads to conservation of the Bernoulli quantities and (zero) vorticity along each streamline and indeed 
to Laplace's equation for the scaled stream function. The flow enters and leaves the present Euler region unidirectionally but with an overall displacement of its streamlines accompanied by pressure changes, inside each gap, consistent with the upstream-to-downstream jumps described above, and with smooth attached flow in between such that the stream function is an unknown constant on each of the quasi-semi-infinite grains. Relatively thin viscous boundary layers are generated of course on every local leading-edge surface here, and these are supposed to remain broadly attached, for some grain surface shapes at least (with local rounding, say), before forming the beginnings of a relatively small Blasiuslike effect on each grain over the longer scale of (2.3a)-(2.3f). Substantial leading-edge separations are thus discounted.

We assume also that no substantial flow separation takes place anywhere. On the longer major length scale of (2.3a)-(2.3f) the flow therefore remains irrotational to leading order virtually everywhere in the flow field and the scaled vorticity is identically zero. Hence the thin-layer scalings above yield the requirement that $u_{n}=u_{n}(x, t)$ must be independent of $\hat{y}$. It follows then from (2.3a) that $\hat{v}_{n}$ varies linearly between its values on the $n$th and $(n-1)$ th grain surface. So the equations of motion within the fluid gaps become

$$
\begin{gathered}
\partial\left(f_{n}-f_{n-1}\right) / \partial t+\partial\left(u_{n}\left(f_{n}-f_{n-1}\right)\right) / \partial x=0, \\
\partial u_{n} / \partial t+u_{n} \partial u_{n} / \partial x=-\partial p_{n} / \partial x,
\end{gathered}
$$

where the influence of the unknown gap width $\left(f_{n}-f_{n-1}\right)$ now shows up. The Euler jumps local to the leading edges also impose the constraints

$$
p_{n}+\frac{1}{2} u_{n}^{2}=\frac{1}{2} \text { at } x=0+,
$$

whereas the Kutta conditions at the successive trailing edges yield

$$
p_{n}=\pi_{e}(t) \text { for all } n \text { at } x=1-,
$$

with the unknown downstream pressure level $\pi_{e}$ being independent of $n$. If for convenience we also define $f_{0}=0, f_{N}=1$ for the side wall surfaces at all $t$ then the overall mass-conservation balance requires

$$
\sum_{1}^{N} u_{n}\left(f_{n}-f_{n-1}\right)=1 \quad \text { at } x=1-,
$$

in view of the incident conditions ahead of the array of grains. Equations (2.4a)(2.4e) describe the fluid-dynamical part of the interactive motion.

In the solid-body part of the motion each grain is driven predominantly here by the fluid-dynamical pressure forces acting laterally on either of its surfaces. At this stage it is useful to be more explicit for thin grains which are also straight (zero camber). The grain positions can then be specified simply by

$$
f_{n}(x, t)=h_{n}(t)+\left(x-\frac{1}{2}\right) \theta_{n}(t),
$$

with $h_{n}, \theta_{n}$ giving the midpoint of the $n$th grain and the grain's angle of inclination in turn and being unknown functions of time $t$; see Figure 1(c) again. The midpoint positions are the centres of mass for grains which, as here, have 
uniform density distribution. In consequence the equation of lateral motion for each grain takes the form

$$
M_{n} d^{2} h_{n} / d t^{2}=\int_{0}^{1}\left(p_{n}-p_{n+1}\right) d x .
$$

Here $M_{n}$ is the scaled mass of the $n$th grain per unit width normal to the $x-$ $y$ plane, given by $M_{n} \equiv M_{n}^{*} \beta /\left(\rho^{*} L_{1}^{* 2}\right)$ where the dimensional grain mass $M_{n}^{*}$ might vary over the $(N-1)$ grains and the relations (2.1a) and (2.1b) are taken into account. Similarly, the equation of angular motion of each grain gives

$$
I_{n} d^{2} \theta_{n} / d t^{2}=\int_{0}^{1}\left(x-\frac{1}{2}\right)\left(p_{n}-p_{n+1}\right) d x,
$$

where the scaled moment of inertia $I_{n} \equiv I_{n}^{*} \beta /\left(\rho^{*} L_{1}{ }^{* 4}\right)$ and $I_{n}^{*}$ is the dimensional moment of inertia of the $n$th grain. Clearly the nondimensionalization here, compared with that on the mass $M_{n}$ above, is in keeping with the moment of inertia being the product of the mass and the square of a radius of inertia. The central case of $\left(M_{n}, I_{n}\right)=(M, I)$ being independent of $n$ will be our concern here.

The system controlling the grain-fluid interactions is $(2.4 \mathrm{a})-(2.4 \mathrm{e}),(2.5 \mathrm{a})-$ (2.5c) subject to suitable initial conditions, with $n$ running from 1 to $N$ (the number of gaps) in (2.4a)-(2.4d) and from 1 to $N-1$ (the number of grains) in $(2.5 \mathrm{a})-(2.5 \mathrm{c})$. The unknown fluid pressures and longitudinal velocities $p_{n}$, $u_{n}$ respectively for $n=1$ to $N$ depend on $x, t$, and the trailing-edge pressure $\pi_{e}$ is also unknown at each time $t$, while the unknown grain midpoint positions and angles $h_{n}, \theta_{n}$ respectively for $n=1$ to $N-1$ also depend on the scaled time $t$ alone. Thickness and camber can readily be incorporated as indicated after (2.3e). Approximate orders of magnitude are also noteworthy in that from (2.4b) the pressure $p$ is of order $u^{2}$ which is $O\left(t^{-2}\right)$ from (2.4a) and (2.4b) since $x$ is typically of order unity. Accordingly both sides of (2.5b) and (2.5c) balance as $O\left(t^{-2}\right)$, a feature which is accommodated in the study in the next section and agrees with an added-mass interpretation used in $\S 4$. This is refined in $\S \S 4,5$ where the balance just mentioned no longer holds.

We comment finally here on Figure 1(b) again in which leading edges and trailing edges are not aligned. As an Euler jump occurs at each such leading edge and an equi-pressure requirement holds at each trailing edge, the second group in the figure has no effect on the first. Further, the dynamics of the first group is very similar to that of an aligned group highlighted in the present work.

\section{$\S 3$. Linear and nonlinear behaviour.}

3.1. Nonlinear study and solutions. The nonlinear behaviour of the grainsfluid system was investigated through numerical studies based on finite differencing as follows. For the current flat-plate cases numerical and analytical treatments were possible in parts of the scheme and their results agreed. We present the numerical treatment because of its wider potential use.

Initial values denoted by a superscript (0) are set for the arrays $p_{n}, u_{n}, h_{n}, \theta_{n}, d h_{n} / d t, d \theta_{n} / d t$. To advance by a specified small time step $\delta t$ to 
the next time stage an estimate is first made for the scaled trailing-edge pressure level $\pi_{e}$. Then for each $n$, with a specified small spatial step $\delta x,(2.4 \mathrm{a})$ is treated in the discretized form

$$
\begin{aligned}
& {\left[H_{n}+\left(\alpha_{n-1}-\alpha_{n}\right)\left(x-\frac{1}{2}\right)\right] u_{n i}} \\
& \quad=\left[c_{n}-\left(\frac{d H_{n}}{d t}\right)\left(x-\frac{1}{2}\right)-\frac{1}{2}\left(\frac{d \alpha_{n-1}}{d t}-\frac{d \alpha_{n}}{d t}\right)\left(x-\frac{1}{2}\right)^{2}\right],
\end{aligned}
$$

and solved for the $u_{n}=u_{n i}$ values at the next time stage from $i=1$ to $\hat{I}(x=0$ to 1) where $x=(i-1) \delta x$ and $(\hat{I}-1) \delta x=1$. Here $H_{n}=h_{n}^{(0)}-h_{n-1}^{(0)}$ is the scaled gap width at the midway station, $\alpha_{n}=-\theta_{n}^{(0)}$, and similarly for the temporal derivatives, while $c_{n}$ follows from the starting value $u_{n 1}$ of $u_{n}$ at $x=0$ which is guessed, corresponding to a guess for the value of the scaled pressure $\pi_{n}$ at the leading-edge station in view of (2.4c). The $c_{n}$ values actually give the mass flux at the station of the centre of mass. Further, the grain-position dependence of (2.5a) is clearly incorporated in (3.1a) and below. Next (2.4b) is discretized as

$$
\left(q_{n i}-q_{n i-1}\right) / \delta x=-\left(u_{n i-1}-u_{n i-1}^{(0)}\right) / \delta t,
$$

for $i \geq 2$, where $p_{n} \equiv \frac{1}{2}\left(1-u_{n}^{2}\right)+q_{n}$ and $q_{n}$ is zero at the leading edge from (2.4c). This enables the $q_{n i}$ to be determined from $i=1$ to $\hat{I}$ and thus the $p_{n i}$ values are determined. The constraint (2.4d) is then addressed. Iterations are performed to update the starting value $u_{n 1}$ (and hence $c_{n}$ and $\pi_{n}$, given by $\left.\frac{1}{2}\left(1-u_{n 1}^{2}\right)\right)$, re-solve (3.1a) and (3.1b) for the velocities and pressures, examine the difference between the trailing-edge pressure $p_{n I}$ and the estimated level $\pi_{e}$, and continue iterating until that difference is suitably small. The procedure is carried out over all the $n$ values, taking account of the fixed values of $f_{0}, f_{N}$, at which stage in effect (2.4a)-(2.4d) are satisfied in discrete form for all $n=1$ to $N$. The summation on the left-hand side in (2.4e) is then done, including all the downstream-end values $u_{n} \hat{l}$; this summation is equivalent to $\sum c_{n}$, from (3.1a). The sum found is generally different from the value of unity required by the right-hand side, and so iterations are made of the pressure level $\pi_{e}$ in order to reduce the left-right difference in (2.4e) to within a very small tolerance.

To complete the description of the time stage, $(2.5 \mathrm{~b})$ is integrated to give the updated $d h_{n} / d t$ and $h_{n}$ arrays, for all $n$, using the pressure distributions worked out as in the previous paragraph. Likewise (2.5c) is integrated for $d \theta_{n} / d t$ and then $\theta_{n}$ for all $n$, following which the coefficients in (3.1a) can be updated to start another iteration, until convergence. The same approach is used to advance to the next time stage, with the $p_{n i}{ }^{(0)}$ being updated to $p_{n i}, h_{n}^{(0)}$ to $h_{n}$, and so on through subsequent time stages. The treatment coped with the added-mass effect, covered a significant range of $(M, I)$ values, and used typical time steps $\delta t$ and spatial steps $\delta x$ of $10^{-4}-10^{-5}$ and 0.005 in turn for most cases considered.

The results are presented in Figures 2-7. See also [4] on fluttering leaves and [52] on flapping flags. Figures 2 and 3 show the solutions obtained for $N=2$, 

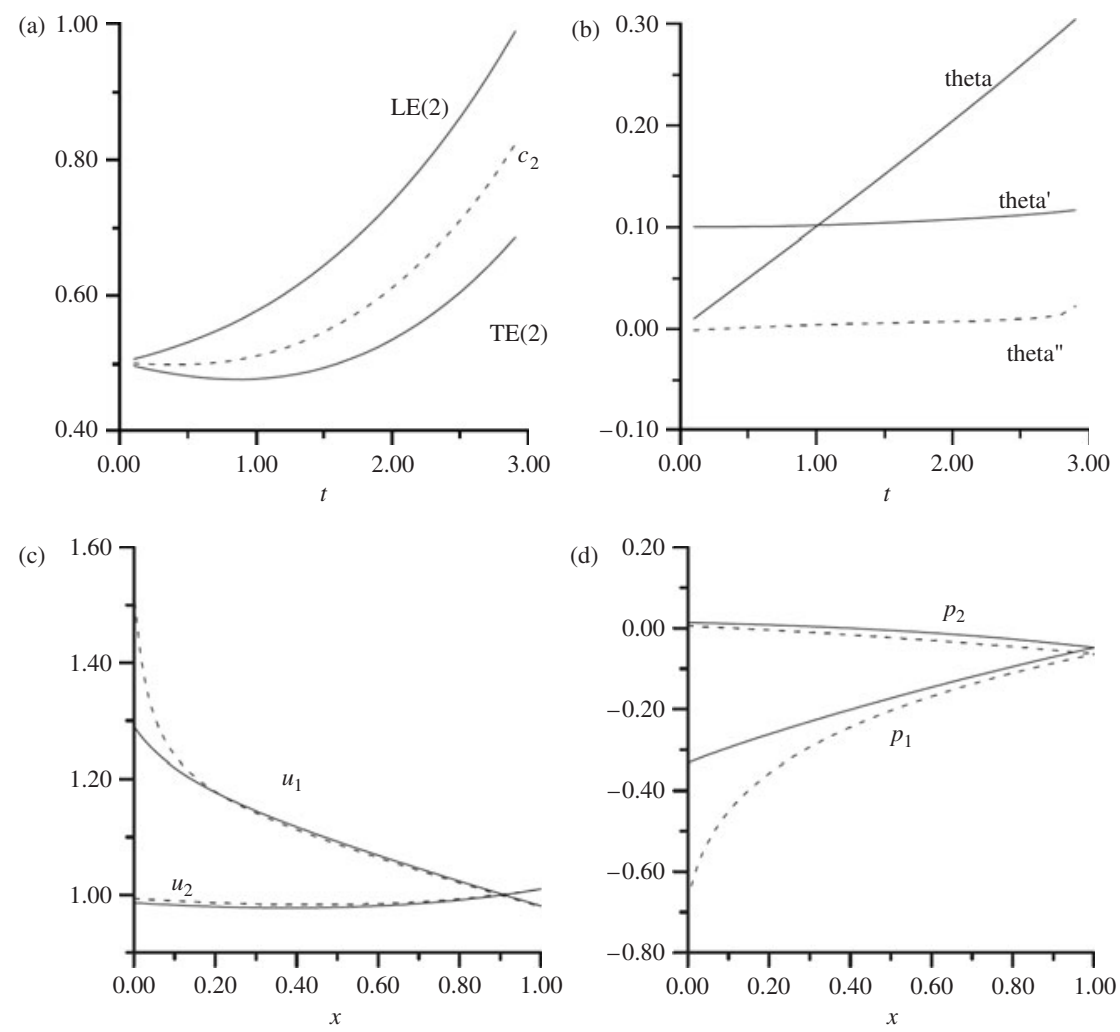

Figure 2: Results for $N=2$ (1 grain) when $(M, I)$ is $(1,2)$ : (a) positions, mass flux; (b) angles; (c) velocity profiles; (d) pressure.

i.e. for a single grain, under different specific conditions. The former figure is for an $(M, I)$ pair of $(1,2)$ with $f_{1}$ equal to $1 / 2$ initially while $d \theta_{1} / d t$ is 0.1 and $c_{1}$ is $1 / 2$ initially. Figure 2(a) shows the evolution of $1-f_{1}$ at the leading and trailing edges ( $L E(2), T E(2)$ respectively) and $c_{2}$, while Figure 2(b) shows $\theta, d \theta / d t, d^{2} \theta / d t^{2}$, with $d \theta / d t$ remaining almost constant until near the end of the calculation run, and Figure 2(c) and (d) present the velocity and pressure profiles $\left(u_{1}, u_{2}, p_{1}, p_{2}\right)$ towards the end of the run. Figure 3 has the same quantities plotted and the same initial conditions but with $(M, I)$ equal to $(10,20)$. We recall that the gap widths are $f_{1},\left(1-f_{1}\right)$ for $N=2$. In all cases studied, including those presented in the figures, the motion of the grain and the surrounding fluid seems to continue smoothly until the gap width at some location approaches zero. The common location is the leading edge of the grain. The time involved appears to be finite on the present scale according to checks made on the accuracy of the solution, indicating the possibility of a finite-time clash or an approach to such a clash arising. During the onset of an apparent clash the velocities within the closing gap become relatively large, as might be expected physically, and likewise for the pressure magnitudes.

Figure 4 then presents results obtained for $N=2$ with an $(M, I)$ of $(1,1)$ but with a relatively small initial disturbance to uniform flow as the starting condition 
(a) 0.

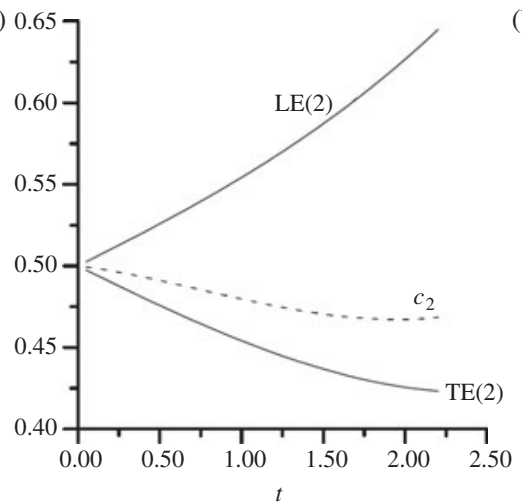

(c) 20.00

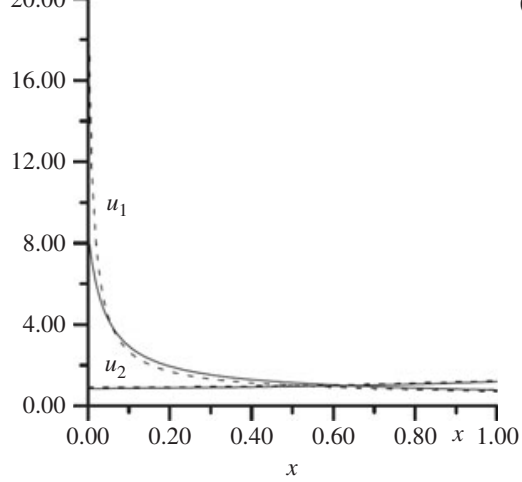

(b)

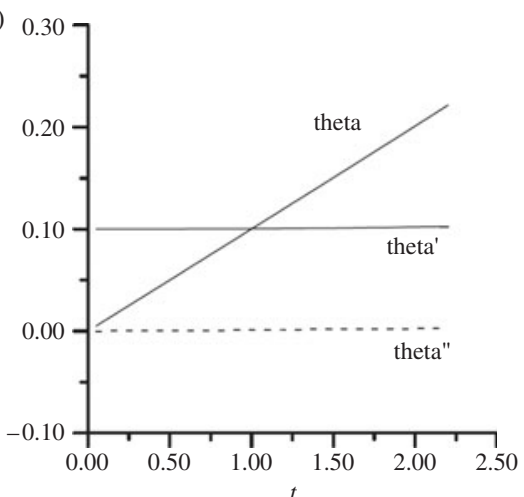

Figure 3: Results for $N=2$ for an $(M, I)$ pair of $(10,20)$.

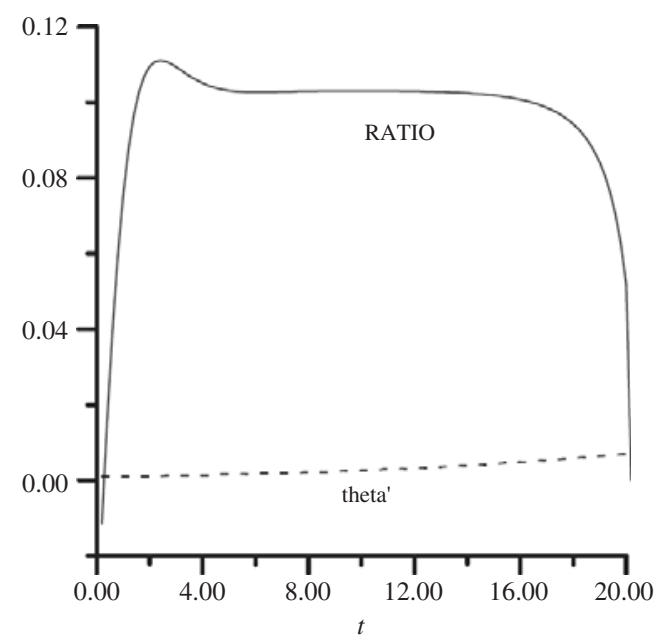

Figure 4: Results for $N=2$ with a small initial disturbance to a uniform state. RATIO denotes $d^{2} \theta / d t^{2}$ divided by $d \theta / d t$. 

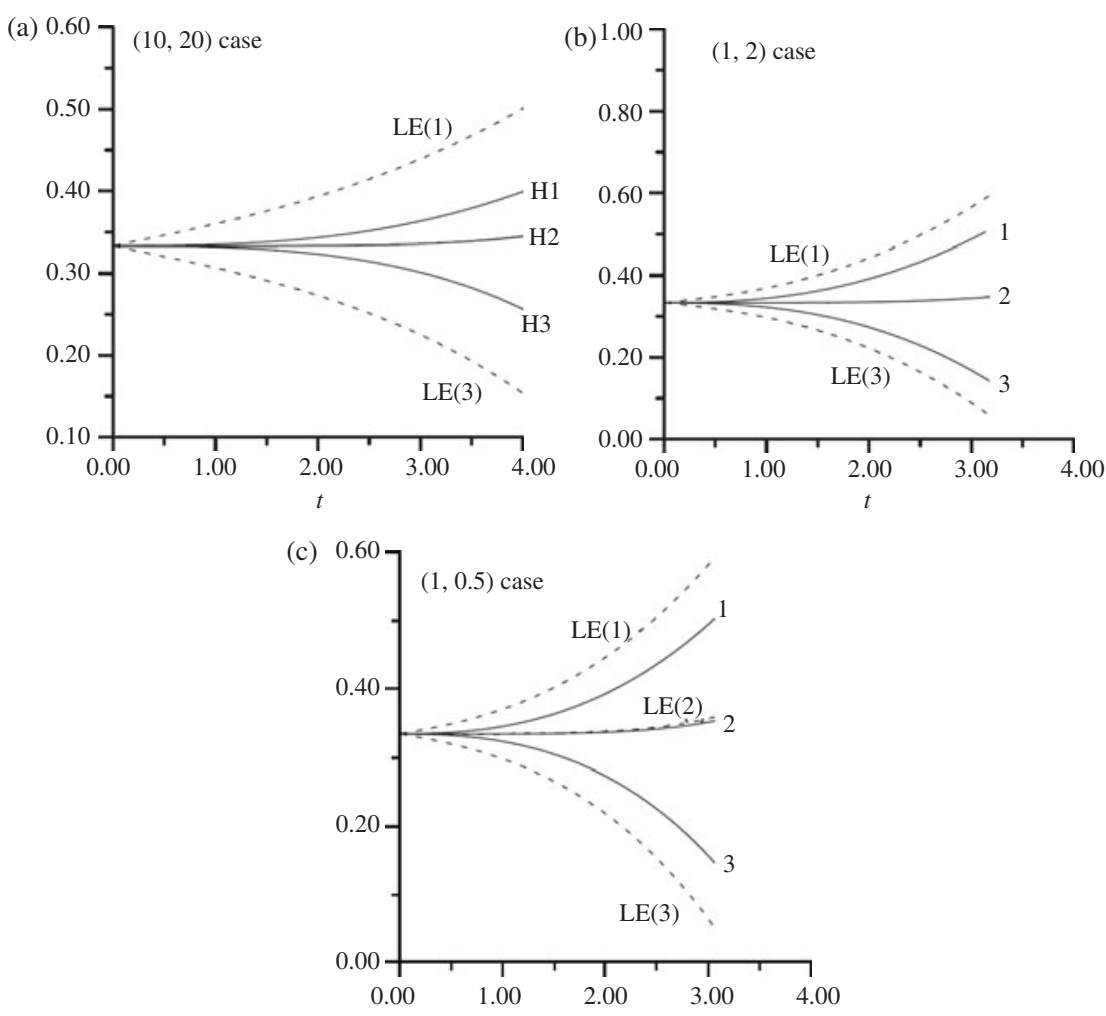

Figure 5: Solutions when $N=3$ for three different values of $(M, I)$.
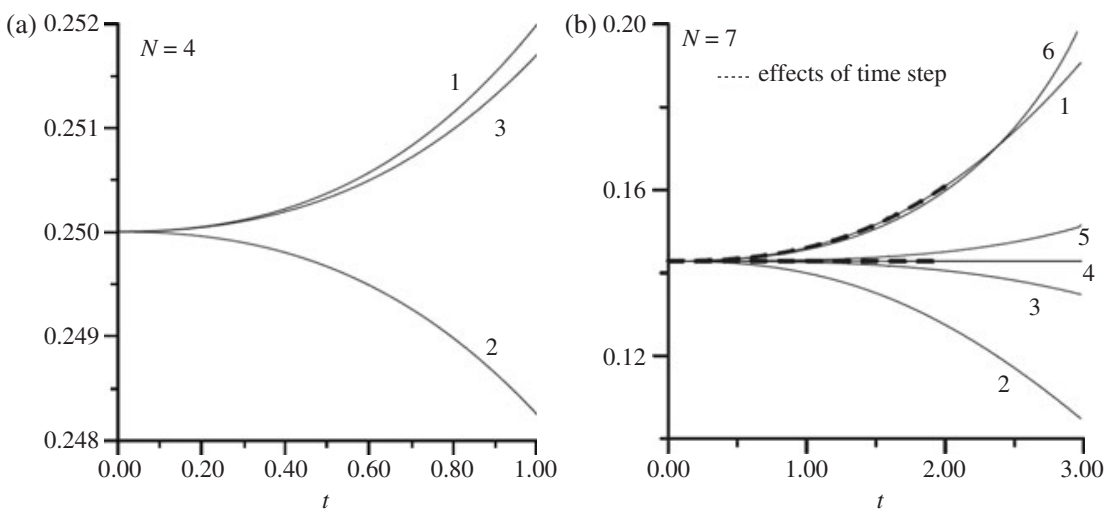

Figure 6: Solutions when $N=4,7$. 

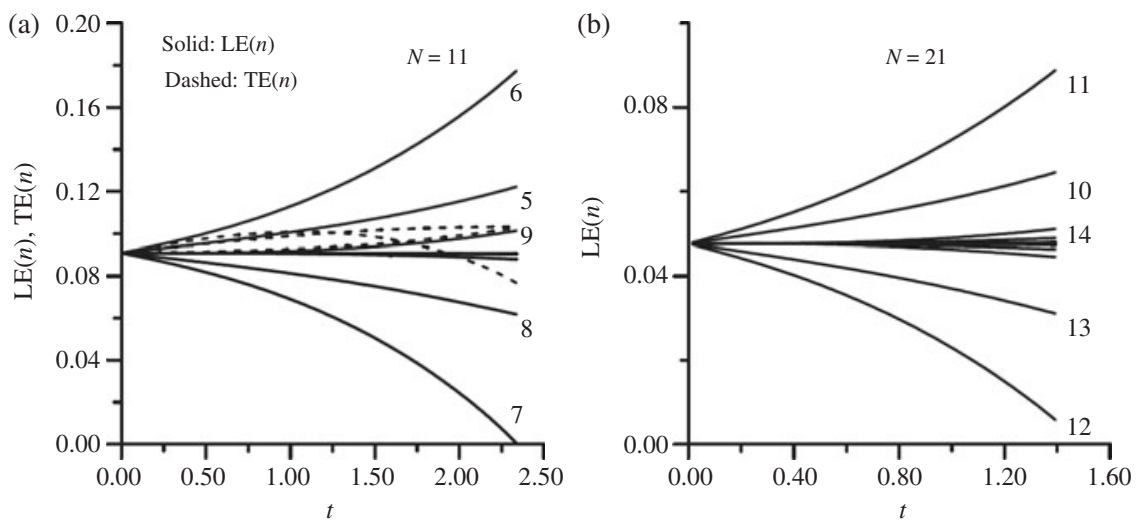

Figure 7: Solutions when $N=11,21$.

at time zero. This is indicated in the plot of $d \theta / d t\left(\theta^{\prime}\right)$, while also shown (as RATIO) is the evolution of $\left(d^{2} \theta / d t^{2}\right) /(d \theta / d t)$. An extended period of growth of the disturbance is observed, and it appears to take an exponential form (since RATIO is nearly constant then), until significant nonlinear effects come into action quite late on in the evolution and these lead on into behaviour similar to that described for the previous two figures. Here and in all the other Figures 2, 3, 5-7 there seems to be an implied self-supporting interplay between the fluid and the grain motion. In such interplay, approximately, a grain movement involving a slight increase in the gap width (or a rotation) on one side of the grain raises the fluid-induced pressure on that side, and correspondingly reduces the pressure on the opposite side, thereby causing a reinforcement of the grain movement, and so on. This interplay in the present inviscid-dominated interactions (it tends to be absent in viscous-dominated settings) will be investigated analytically in the next subsection.

The results in Figure 5 are for $N=3$ and in Figure 6 are for $N=4$ and $N=7$. These and related results show that the trends suggested in the earlier figures for $N=2$ tend to continue for higher values of $N$. Small initial disturbances are found to grow in amplitude with increasing time, pointing to nonlinear interaction as being inevitable, and such interaction then eventually produces a solid-to-solid clash within a finite time at a leading-edge location, according to all the studies made. Figure $5(\mathrm{a}-\mathrm{c})$ also covers three different combinations of $(M, I)$ values, namely $(10,20),(1,2)$ and $(1,1 / 2)$, for each of which the evolutionary trends are the same at heart; the quantities $H 1, H 2, \ldots$ or $1,2, \ldots$ refer to the respective gap widths at the midway station. Figure 6(b) also illustrates the typical effects of halving the time step, which are tiny, as are those of the spatial step.

Further increased values of $N$, namely 11 and 21, yield the results given in Figure 7. Again the earlier mentioned trends of growth and clashing tend to be confirmed, with one leading-edge gap in particular apparently approaching zero in a finite time for each case. In addition, however, the cases of 11 and 21 indicate that a large- $N$ limit may be showing signs of emerging as the results 
for the two cases, started from broadly the same initial conditions, are somewhat similar in terms of the evolution viewed over the large spatial scale in Figure 7.

The nonlinear behaviour shown highlights three major features above all, namely an apparent growth of small disturbances, an apparent clashing of a grain with a neighbouring grain or with a side wall within a finite time, and the issue of what happens if there are many grains in the system. These features are discussed in the next subsection, in $\$ \S 4$ and 5 respectively, with methods covering any $(M, I)$ values.

3.2. Linear behaviour. Small disturbances from a uniform state, say for relatively early times, yield useful analytical insights as well as checks here. The simplest uniform state is for a single thin straight grain and two equal gaps for which $N=2, f_{0}=0, f_{2}=1, h_{1}=\frac{1}{2}, \theta_{1}=0$, and $u_{n}=1, p_{n}=0$ for $n=1,2$. Small disturbances in the form $h_{1}=\frac{1}{2}+\tilde{h} \exp (Q t), \theta_{1}=\tilde{\theta} \exp (Q t)$ and so on, with explicit time dependence as shown, can then be considered.

Omitting details, it is found that the eigenrelation for the unknown constant $Q$ is

$$
\begin{gathered}
-(3 M+1)\left(I+\frac{1}{180}\right) Q^{5}-\left(3 M I+\frac{M}{10}+4 I+\frac{1}{20}\right) Q^{4} \\
+\left(\frac{M}{2}-6 I-\frac{1}{5}\right) Q^{3}+\left(M-\frac{1}{3}\right) Q^{2}=0 .
\end{gathered}
$$

This polynomial equation has two trivial equal roots $Q=0$ which correspond to a uniform translation of the grain. The values of the other three roots (say, $Q_{1}, Q_{2}, Q_{3}$ ) depend on the parameters $M, I$ as studied in Figure 8. Although the figure deals with an extensive range of $(M, I)$ values for completeness, the trend is quite clear. Whenever $M>\frac{1}{3}$ there is a single positive real root $Q_{3}$ whereas the other two roots $Q_{1}, Q_{2}$ are either real and negative or complex conjugate values with a negative real part. So the dominant root $Q_{3}$ indicates instability in the sense of exponential growth of the disturbance in time, while the roots $Q_{1}, Q_{2}$ yield temporal stability. There are other conditions on $M, I$ which affect the appearance of an unstable mode or not, but the criterion $M>\frac{1}{3}$ is the major one.

The dominant root from (3.2) yields good agreement with the growth seen in the nonlinear numerical results earlier for specific values of $M, I$. In particular for $(M, I)$ equal to $(1,1)$ the predicted $Q_{3}$ value from (3.2) is 0.103 ; this value is very close to the growth rate (denoted RATIO) found in the computed nonlinear case of Figure 4 at low amplitudes. We shall return in $\$ 5$ to the case of unequal gaps as well as to the properties of small disturbances for many gaps.

§4. Clashes of grains. A "clash" appears to be the typical final outcome of the nonlinear results above, in which the scaled gap width tends to zero. We note previous numerical studies of other unsteady-flow configurations $[2,17,23,58]$ which have pointed to finite-time clashing, in contrast with steady-flow studies of ground effects $[\mathbf{2 8}, \mathbf{5 1}]$ for example. A description of a finite-time clash as 


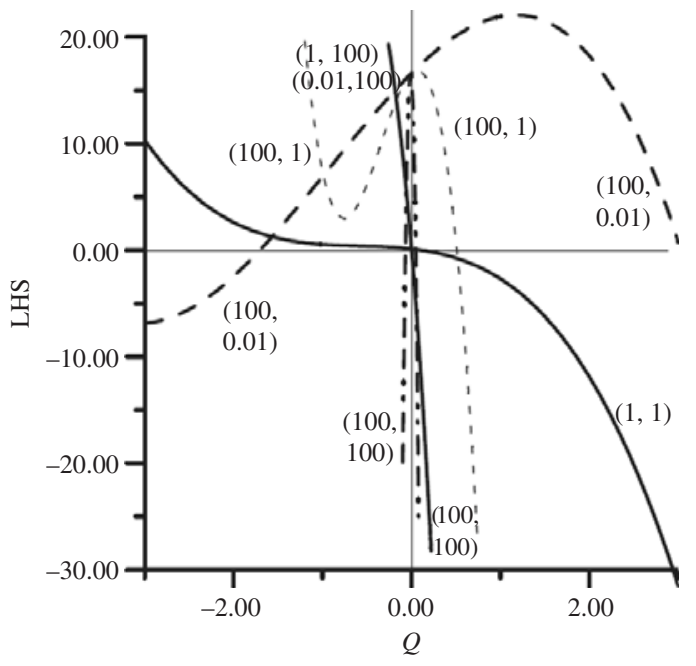

Figure 8: The left-hand side LHS of the polynomial equation (3.2) (divided by $Q^{2}$ ) for various $(M, I)$ pairs.

guided by the computational results is now sought analytically for the case of a single grain (a straight thin body) along with gaps labelled 1, 2, with a view to extending it afterwards to the multi-grain regime. Thus, based on (2.4a)-(2.5c), the system to be addressed is

$$
\begin{gathered}
u_{1}=\frac{\left[c_{1}-\left(x-\frac{1}{2}\right) \dot{h}_{1}-\frac{1}{2}\left(x-\frac{1}{2}\right)^{2} \dot{\theta}_{1}\right]}{\left[h_{1}+\left(x-\frac{1}{2}\right) \theta_{1}\right]}, \\
u_{2}=\frac{\left[1-c_{1}+\left(x-\frac{1}{2}\right) \dot{h}_{1}+\frac{1}{2}\left(x-\frac{1}{2}\right)^{2} \dot{\theta}_{1}\right]}{\left[1-h_{1}-\left(x-\frac{1}{2}\right) \theta_{1}\right]}, \\
\frac{\partial u_{n}}{\partial t}+u_{n} \frac{\partial u_{n}}{\partial x}=-\frac{\partial p_{n}}{\partial x}, \\
p_{n}+\frac{1}{2} u_{n}^{2}=\frac{1}{2} \quad \text { at } x=0, \\
p_{1}=p_{2} \text { at } x=1, \\
M \ddot{h}_{1}=\int_{0}^{1}\left(p_{1}-p_{2}\right) d x, \\
I \ddot{\theta}_{1}=\int_{0}^{1}\left(x-\frac{1}{2}\right)\left(p_{1}-p_{2}\right) d x,
\end{gathered}
$$

where a dot denotes the ordinary derivative $d / d t$, while $u_{n}, p_{n}$ are unknown functions of $x, t$ and $n=1,2$ in (4.1c) and (4.1d). The properties

$$
f_{1}=h_{1}(t)+\left(x-\frac{1}{2}\right) \theta_{1}(t), \quad c_{1}+c_{2}=1, \quad f_{0}=0, \quad f_{2}=1
$$

have been used above, while as mentioned earlier (4.1c) and so on can be integrated analytically but the existing forms seem to yield a clearer picture. 
The response as $t \rightarrow t_{0}-$ is of interest here, with $t_{0}$ being a finite scaled time at which without loss of generality the gap width $h_{1}+\left(x-\frac{1}{2}\right) \theta_{1}$ becomes small at some point, specifically at the leading edge $x=0$.

An argument can be made first for an approach to a similarity form as $t \rightarrow t_{0}-$ in which $h_{1}-h_{1}^{(0)}, \theta_{1}-\theta_{1}^{(0)}$ are of order $\left(t_{0}-t\right)^{\sigma}$, say, with the power $\sigma$ being positive and with the limiting values (constants) $h_{1}^{(0)}, \theta_{1}^{(0)}$ satisfying

$$
h_{1}^{(0)}=\frac{1}{2} \theta_{1}^{(0)} .
$$

Equation (4.2) corresponds to a clash at the leading edge $x=0+$. The inferred size for $c_{1}$ is then of order $\left(t_{0}-t\right)^{\sigma-1}$ however, from the numerator in (4.1a), and so then the numerator in (4.1b) suggests the value $\sigma=1$; this is in order to prevent $u_{1}, u_{2}$ being of opposite sign to each other if $\sigma<1$, hence provoking unrealistic reversed flow, and also to allow $c_{1}$ to have a substantial role. Here the condition $\theta_{1}^{(0)}<1$ is assumed which implies that gap 2 remains open everywhere whereas gap 1 is closing at the leading edge. The expansion called for then has

$$
\begin{gathered}
\left(h_{1}, \theta_{1}\right)=\left(h_{1}^{(0)}, \theta_{1}^{(0)}\right)+\left(t_{0}-t\right)\left(h_{1}^{(1)}, \theta_{1}^{(1)}\right)+\Delta\left(h_{1}^{(2)}, \theta_{1}^{(2)}\right)+\cdots \\
c_{1}=c_{1}^{(1)}+\dot{\Delta} c_{1}^{(2)}+\cdots
\end{gathered}
$$

with the function $\Delta(t)$ to be found and such that $\Delta \ll\left(t_{0}-t\right)$ in view of the ordering in (4.3a). It follows that the velocities are given by

$$
\begin{gathered}
u_{1}=\frac{\left[\left\{c_{1}^{(1)}+\left(x-\frac{1}{2}\right) h_{1}^{(1)}+\frac{1}{2}\left(x-\frac{1}{2}\right)^{2} \theta_{1}^{(1)}\right\}+\dot{\Delta}\left\{F+G x+H x^{2}\right\}+\cdots\right]}{\left[x \theta_{1}^{(0)}+\left(t_{0}-t\right)\left\{h_{1}^{(1)}+\left(x-\frac{1}{2}\right) \theta_{1}^{(1)}\right\}+\cdots\right]}, \\
u_{2}=\frac{\left[\left\{1-c_{1}^{(1)}-\left(x-\frac{1}{2}\right) h_{1}^{(1)}-\frac{1}{2}\left(x-\frac{1}{2}\right)^{2} \theta_{1}^{(1)}\right\}+\dot{\Delta}\left\{-F-G x-H x^{2}\right\}+\cdots\right]}{\left[\left\{1-x \theta_{1}^{(0)}\right\}+\left(t_{0}-t\right)\left\{-h_{1}^{(1)}-\left(x-\frac{1}{2}\right) \theta_{1}^{(1)}\right\}+\cdots\right]},
\end{gathered}
$$

from (4.1a) and (4.1b), while (4.1c) and (4.1d) then yield the induced pressures as

$$
p_{n}=\frac{1}{2}\left(1-u_{n}^{2}\right)-\int_{0}^{x} \frac{\partial u_{n}}{\partial t} d x
$$

for $n=1,2$. The integral in $(4.4 \mathrm{c})$ acts as the temporal derivative of a velocity potential. The constants $F, G, H$ are of order unity, $F$ being the combination $c_{1}^{(2)}+\frac{1}{2} \dot{\Delta} h_{1}^{(2)}-\frac{1}{8} \dot{\Delta} \theta_{1}^{(2)}$ while $G, H$ are linearly dependent on $h_{1}^{(2)}, \theta_{1}^{(2)}$. Also we observe that the leading terms in $u_{1}, u_{2}$ for $x$ of $O(1)$ are independent of time $t$. The properties in gap 1 are of most immediate concern as they show the existence of not only global effects associated with $x \sim 1$ but also a local region near the leading edge in gap 1 where $x$ is of order $t_{0}-t$ because of the denominator containing $x \theta_{1}^{(0)}$ in (4.4a). Such a leading-edge effect is absent in the gap 2 behaviour since there the contribution $\left\{1-x \theta_{1}^{(0)}\right\}$ remains of order unity throughout. The pressure response and hence grain motion associated with 
gap 1 can be deduced from (4.4a) and (4.4c) in composite form by allowing for the two streamwise length scales involved. It is taken of course that $h_{1}^{(1)}>\frac{1}{2} \theta_{1}^{(1)}$ which corresponds to the gap width being positive rather than negative just prior to the clash. The properties in gap 1 also require the relation

$$
c_{1}^{(1)}-\frac{1}{2} h_{1}^{(1)}+\frac{1}{8} \theta_{1}^{(1)}=0
$$

to hold, which has the effect of keeping $u_{1}$ finite as $x$ tends to zero in the leading terms in $u_{1}$ just mentioned. The reason for this, in brief, is that otherwise $u_{1}$ would be of order $1 /\left(t_{0}-t\right)$ in the leading-edge region, making $p_{1}$ of the same order for all $x$ of $O(1)$ including the trailing-edge station and mismatching with the pressure response $p_{2}$ there.

Seeking a first possible term which is non-zero in the fluid acceleration $\partial u_{1} / \partial t$ as $t \rightarrow t_{0}-$ near the leading edge, we anticipate that $\dot{\Delta}$ is small (essentially repeating the condition stated after (4.3b)) but exceeds $O\left(t_{0}-t\right)$. Hence (4.4a) yields a dominant contribution, with $\lambda$ denoting $\left(h_{1}^{(1)}-\frac{1}{2} \theta_{1}^{(1)}\right) / \theta_{1}^{(0)}$ which is positive,

$$
\frac{\partial u_{1}}{\partial t}=\left\{\frac{F}{\theta_{1}^{(0)}}+O(x)\right\} \partial_{t}\left[\frac{\dot{\Delta}}{x+\lambda\left(t_{0}-t\right)+O\left(x\left(t_{0}-t\right)\right)}\right] .
$$

The pressure response $p_{1}$ in (4.4c) for $n=1$ is therefore mainly from the integral part which gives

$$
p_{1}=-\left\{\frac{F}{\theta_{1}^{(0)}}+O(x)\right\} \partial_{t}\left[\dot{\Delta} \ln \left(\frac{\lambda^{-1} x+t_{0}-t}{t_{0}-t}\right)\right],
$$

in contrast with the localized part $\frac{1}{2}\left(1-u_{1}^{2}\right)$ which is of order unity because of (4.4a) combined with (4.5). Similarly, the fluid acceleration $\partial u_{2} / \partial t$ throughout gap 2 has the form of an $O(1)$ function of $x$ multiplied by $\ddot{\Delta}$ in view of (4.4b). In consequence the induced pressure $p_{2}$ in gap 2 comes from the integrated unsteady part in (4.4c) with $n=2$, in the main, giving

$$
p_{2}=O(\ddot{\Delta})+O(1)
$$

in gap 2. The trailing-edge condition (4.1e) now requires the expressions in (4.6b) and (4.6c) to be equal at $x=1$ and so, given the logarithmic factor in (4.6b), $\dot{\Delta} \ln \left(t_{0}-t\right)$ must be constant to leading order. Accordingly the result

$$
\begin{gathered}
\dot{\Delta}=-\left(\ln \left(t_{0}-t\right)\right)^{-1}+O(1), \\
\Delta=\left(t_{0}-t\right)\left\{\left(\ln \left(t_{0}-t\right)\right)^{-1}+O(1)\right\}
\end{gathered}
$$

applies without loss of generality for the temporal dependence as $t \rightarrow t_{0}-$. A constant multiplicative factor of order unity could be inserted in (4.7a) and (4.7b) but instead this is taken to be absorbed into the corresponding coefficients in (4.3a), (4.3b), (4.4a) and (4.4b). The earlier suppositions that $\Delta \ll\left(t_{0}-t\right)$ and $\dot{\Delta} \gg\left(t_{0}-t\right)$ are seen to be borne out by (4.7a) and (4.7b), as a check. 
Turning to the grain motion produced, we have the left-hand side of (4.1f) being of size $O(\ddot{\Delta})$ from the expansion (4.3a) whilst the right-hand side is dominated by the integral of $p_{1}$ over all $x$ from comparing the pressures in (4.6b) and (4.6c). Formally the right-hand side is thus the larger. That again necessitates $\dot{\Delta} \ln \left(t_{0}-t\right)$ being constant and so leads to (4.7a) and (4.7b). In (4.1g) likewise the right-hand side overwhelms the left, and $p_{1}$ overwhelms $p_{2}$, yielding (4.7a) and (4.7b) again. This is in effect the added-mass interpretation appearing again but now the actual mass and moment of inertia factors $M, I$ have negligible influence.

The prime predictions for the clash behaviour near $t=t_{0}$ are therefore (4.2), (4.5), (4.7a) and (4.7b) in a sense. Comparisons with the nonlinear numerical solutions presented in $\$ 3$ are fairly supportive of the current predictions.

Quantitative comparisons are given in Figure 9 for a case with one grain and are in terms of the leading-edge grain positions, rotation rates, accelerations, flow velocities, pressures and a number of specific test properties suggested by the analysis just above, such as the relation in (4.5). Figure 9(a) presents the pressure profiles just prior to the envisaged clash, tending to confirm the quite rapid variation in $p_{1}$ compared with that of $p_{2}$, in line with (4.6b) and (4.6c). Figure 9(b) shows the evolution of the leading-edge position $f_{1}(0, t)$, i.e. $h_{1}-\frac{1}{2} \theta_{1}$ as denoted by LE(1), and the left-hand side (LHS) of (4.5); both quantities appear to approach zero as implied by (4.2) and (4.5) in turn. Likewise Figure $9(\mathrm{c})$ indicates that the inverses of the leading-edge velocity $u_{1}(0, t)$ (see 1/LEU) and of the acceleration term $d^{2} h_{1} / d t^{2}$ (see Acceln and 0.01/Acceln) tend slowly to zero in keeping with the predictions in (4.3a) and (4.4a) with (4.7a) and (4.7b).

$\S 5$. Many grains. The interest now is in understanding the combined motion of the fluid and grains when there are a large number of grains, $N \gg 1$, with the typical gap width being small of order $1 / N$. A clue to the interaction structure is then provided by the linear analysis for a single grain as summarized in the second half of $\$ 3$ except with one of the basic gap widths becoming small. It is found that the grain acceleration has negligible influence then and the reduced gap problem becomes a closed one. The same conclusion holds for nonlinear cases.

For the multi-grain scenario the gap width $\left(f_{n}-f_{n-1}\right)$ is expected typically to be of order $N^{-1}$, whereas all other quantities in the controlling equations (2.4a)-(2.4e), (2.5b) and (2.5c) remain of order unity as $N$ becomes large. In detail, $x$ is $O(1)$ in order to cater for the whole grain length, while the characteristic velocity $u$ is envisaged as $O(1)$ to balance the jump effect in (2.4c) at each leading edge, and so the representative time scale might be expected to be of $O(1)$ based on $|x| /|u|$ or on comparing the fluid acceleration and inertia contributions. It follows that the induced pressure $p$ is also generally of order unity in view of (2.4b) and (2.4c) in the fluid. As far as the grain motion is concerned, however, the fact that $\left(f_{n}-f_{n-1}\right)$ is small forces the typical midpoint displacement $h$ and the rotation angle $\theta$ in (2.5a) to remain 

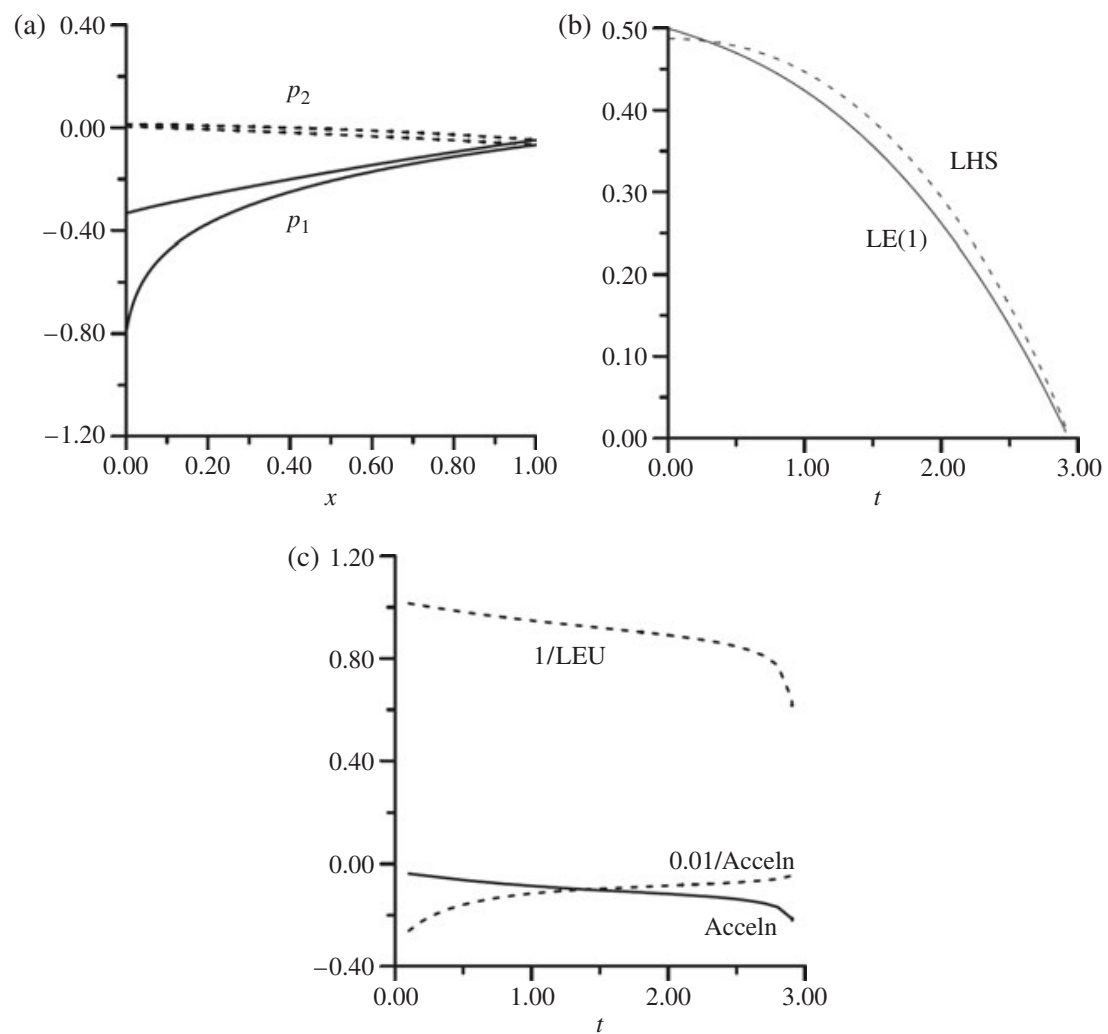

Figure 9: Comparisons between the predictions of the analysis in $\S 4$ for clashes and full evolution results as in $\S 3$.

small, again of the order $N^{-1}$. Hence the acceleration term on the left-hand side of (2.5b) is $O\left(N^{-1}\right)$ in contrast with the right-hand side which is $O(1)$. A similar contrast occurs in $(2.5 \mathrm{c})$. In consequence the contributions from grain acceleration become negligible. The added-mass interpretation is thus similar to that in the previous section.

In the limit, putting $f_{n}-f_{n-1}=R(x, \hat{y}, t) d \hat{y}$ and letting $N$ tend to infinity leads to the continuum equations which are, from substitution into (2.4a), (2.4b), (2.5b) and (2.5c),

$$
\begin{gathered}
\frac{\partial R}{\partial t}+\frac{\partial}{\partial x}(u R)=0, \\
\frac{\partial u}{\partial t}+u \frac{\partial u}{\partial x}=-\frac{\partial p}{\partial x}, \\
0=\frac{\partial}{\partial \hat{y}}\left\{\int_{0}^{1} p(x, \hat{y}, t) d x\right\}, \\
0=\frac{\partial}{\partial \hat{y}}\left\{\int_{0}^{1}\left(x-\frac{1}{2}\right) p(x, \hat{y}, t) d x\right\},
\end{gathered}
$$


in turn, subject to the constraints

$$
\begin{gathered}
\int_{0}^{1} u R d \hat{y}=1 \quad \text { at } x=1, \\
p+\frac{1}{2} u^{2}=\frac{1}{2} \quad \text { at } x=0, \\
p=\pi_{e}(t) \quad \text { at } x=1, \\
\int_{0}^{1} R d \hat{y}=1 \quad \text { for all } x, t,
\end{gathered}
$$

from $(2.4 \mathrm{e}),(2.4 \mathrm{c})$ and $(2.4 \mathrm{~d})$ and the chute shape respectively, and with

$$
R(x, \hat{y}, t)=a(\hat{y}, t)+\left(x-\frac{1}{2}\right) b(\hat{y}, t)
$$

for the main case here of flat grains. Here $a, b$ are the unknown midpointposition and angle functions respectively, $p(x, \hat{y}, t), u(x, \hat{y}, t)$ act in effect as the induced pressure and velocity of the fluid-grain mixture, $R(x, \hat{y}, t)$ acts as an effective shape, slope or transverse density function, while $d \hat{y}$ is essentially a fixed small increment in $\hat{y}$. In the above setting other cases of non-flat grains can be covered by altering the shape dependence in (5.1i) (this aspect is similar to the point made in $\$ 2$ concerning grain thickness effects), while in $(5.1 \mathrm{~g}) \pi_{e}(t)$ denotes the unknown pressure at the beginning of the wake extended from that in $(2.4 \mathrm{~d})$, with the constraint $(5.1 \mathrm{~g})$ being the effective Kutta condition which acts across the whole trailing-edge line. See Figure 10(a). We observe that if the leading- and tailing-edge positions are curved rather than straight as in the figure then the same fluid-grain interaction structure applies. An implicit assumption also in the above is that there are no significant sublayers in terms of $\hat{y}$, for instance near the outer walls.

A study of the full nonlinear system (5.1a)-(5.1i) has still to be carried out, based perhaps on a numerical treatment as in the first half of $\S 3$ and an analytical investigation akin to that in $\$ 4$ concerning clashes within a finite time. Linearized properties are worth considering first here. Since a simple steady solution with uniform motion is $u=1, R=1$ with $p=\pi_{e}=a=b=0$, a perturbation in the form

$$
\left(u, R, p, \pi_{e}, a, b\right)=(1,1,0,0,0,0)+\tilde{\epsilon}\left(\tilde{u}, \tilde{R}, \tilde{p}, \tilde{\pi}_{e}, \tilde{a}, \tilde{b}\right)+\cdots
$$

is considered, where the amplitude $\tilde{\epsilon}$ is small. Substitution into (5.1a)-(5.1i) yields the linear equations

$$
\begin{gathered}
\frac{\partial \tilde{R}}{\partial t}+\frac{\partial \tilde{R}}{\partial x}+\frac{\partial \tilde{u}}{\partial x}=0, \\
\frac{\partial \tilde{u}}{\partial t}+\frac{\partial \tilde{u}}{\partial x}=-\frac{\partial \tilde{p}}{\partial x}, \\
\frac{\partial}{\partial \hat{y}}\left\{\int_{0}^{1} \tilde{p}(x, \hat{y}, t) d x\right\}=0 \\
\frac{\partial}{\partial \hat{y}}\left\{\int_{0}^{1} x \tilde{p}(x, \hat{y}, t) d x\right\}=0,
\end{gathered}
$$


(a)
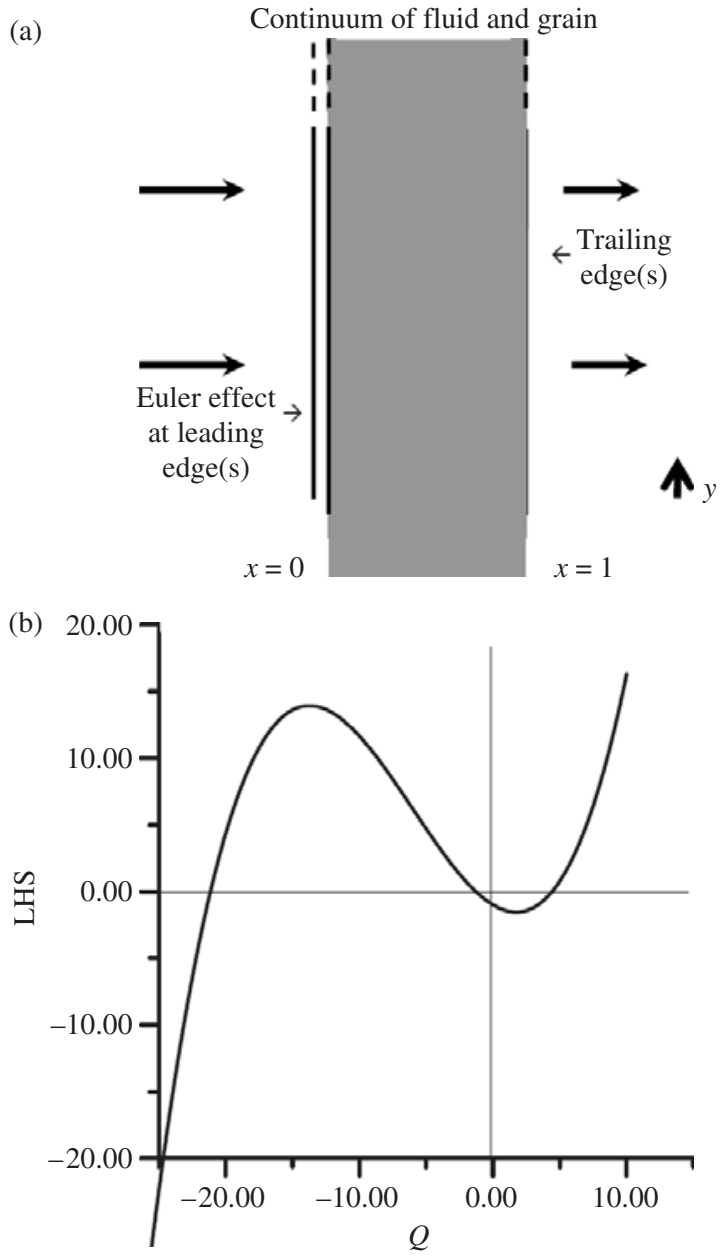

Figure 10: For the continuum limit in §5: (a) a sketch of the interaction structure showing the main continuum region (shaded) and the leading and trailing edges, with or without side walls being present; (b) the left-hand side of (5.8) versus $Q$, indicating the roots $Q_{1}, Q_{2}, Q_{3}$.

together with

$$
\begin{gathered}
\int_{0}^{1}(\tilde{u}+\tilde{R}) d \hat{y}=0 \quad \text { at } x=1, \\
\tilde{p}+\tilde{u}=0 \quad \text { at } x=0, \\
\tilde{p}=\tilde{\pi}_{e}(t) \quad \text { at } x=1, \\
\int_{0}^{1} \tilde{R} d \hat{y}=0 \quad \text { for all } x, t,
\end{gathered}
$$

and for the specific flat case

$$
\tilde{R}=\tilde{a}+\left(x-\frac{1}{2}\right) \tilde{b}
$$


Seeking eigenmodes now we replace $\tilde{R} \longrightarrow \tilde{R} \exp (Q T)$ in the spirit of $\S 3.2$, implying that (5.3a) and (5.3b) are replaced by

$$
\begin{gathered}
Q \tilde{R}+\frac{\partial \tilde{R}}{\partial x}+\frac{\partial \tilde{u}}{\partial x}=0, \\
Q \tilde{u}+\frac{\partial \tilde{u}}{\partial x}=-\frac{\partial \tilde{p}}{\partial x},
\end{gathered}
$$

while (5.3c)-(5.3i) remain unaltered in essence, although the appearance of $t$ is now suppressed. The solution is expressible in terms of the velocity potential $\phi(x, \hat{y})$ such that $\tilde{u}=\partial \phi / \partial x$ and of a mass function $S$ such that $\tilde{R}=\partial S / \partial x$, yielding

$$
\begin{gathered}
\tilde{p}=\tilde{R}+Q(S-\phi), \\
\tilde{u}\left(=\frac{\partial \phi}{\partial x}\right)=-\tilde{R}-Q S,
\end{gathered}
$$

and so

$$
\begin{gathered}
\frac{\partial}{\partial \hat{y}}\left\{\int_{0}^{1}\left(\frac{\partial \phi}{\partial x}+Q \phi\right) d x\right\}=0, \\
\frac{\partial}{\partial \hat{y}}\left\{\int_{0}^{1} x\left(\frac{\partial \phi}{\partial x}+Q \phi\right) d x\right\}=0, \\
\phi(0, \hat{y})=0, \\
\frac{\partial \phi}{\partial x}(1, \hat{y})+Q \phi(1, \hat{y})=\text { constant } \\
\int_{0}^{1} \frac{\partial^{2} \phi}{\partial x^{2}} d \hat{y}=0 .
\end{gathered}
$$

In addition, the specific form (5.3i) requires $\phi$ to be cubic in $x$, from (5.5b), say

$$
\phi=\sum_{k=0}^{3}\left(x-\frac{1}{2}\right)^{k} \hat{A}_{k}(\hat{y}),
$$

for some functions $\hat{A}_{k}$ of $\hat{y}$ to be found. Applying (5.6a)-(5.6d), we find that the four conditions

$$
\begin{gathered}
\hat{A}_{0}-\frac{1}{2} \hat{A}_{1}+\frac{1}{4} \hat{A}_{2}-\frac{1}{8} \hat{A}_{3}=0 \\
Q \hat{A}_{0}+\left(1+\frac{1}{2} Q\right) \hat{A}_{1}+\left(1+\frac{1}{4} Q\right) \hat{A}_{2}+\left(\frac{3}{4}+\frac{1}{8} Q\right) \hat{A}_{3}=\text { constant } \\
Q \hat{A}_{0}^{\prime}+\hat{A}_{1}^{\prime}+\frac{1}{12} Q \hat{A}_{2}^{\prime}+\frac{1}{4} \hat{A}_{3}^{\prime}=0 \\
\frac{1}{12} Q \hat{A}_{1}^{\prime}+\frac{1}{6} \hat{A}_{2}^{\prime}+\frac{1}{80} Q \hat{A}_{3}^{\prime}=0
\end{gathered}
$$

must hold, in the light of (5.6c), (5.6d), (5.6a) and (5.6b) respectively. The constant in (5.7b) is a normalization factor, as is that in (5.6d). The effect of (5.6e) is addressed later. The four conditions serve to control the four unknown functions $\hat{A}_{0}^{\prime}, \hat{A}_{1}^{\prime}, \hat{A}_{2}^{\prime}, \hat{A}_{3}^{\prime}$ self-consistently once (5.7a) and (5.7b) have been differentiated with respect to $\hat{y}$, provided that $Q$ satisfies the 
eigenrelation

$$
\frac{Q^{3}}{120}+\frac{3 Q^{2}}{20}-\frac{3 Q}{5}-1=0 .
$$

The three roots for $Q$ are real, say $Q_{1}, Q_{2}, Q_{3}$, and one of them $Q_{3}$ is positive, giving instability; see Figure 10(b).

The completeness of the solution necessitates checking back, however. For each root $Q,(5.7 \mathrm{a})-(5.7 \mathrm{~d})$ determine $\hat{A}_{1}^{\prime}, \hat{A}_{2}^{\prime}, \hat{A}_{3}^{\prime}$ in terms of the unknown $\hat{A}_{0}^{\prime}(\hat{y})$, say in the form $\hat{A}_{k}^{\prime}=\lambda_{k} \hat{A}_{0}^{\prime}$ for $k=0$ to 3 , with $\lambda_{0}=1$ and with $\lambda_{1}, \lambda_{2}, \lambda_{3}$ being known constants. On integration, therefore,

$$
\hat{A}_{k}(\hat{y})=\lambda_{k} \hat{A}_{0}(\hat{y})+\mu_{k}
$$

for $k=0$ to 3 with $\mu_{0}=0$ and $\mu_{k}$ constants. So (5.7a) and (5.7b) give two equations for the three unknowns $\mu_{1}, \mu_{2}, \mu_{3}$ and the required third equation stems from (5.6e) which has not been used yet but now yields the constraint

$$
\mu_{k}=-\lambda_{k} \int_{0}^{1} \hat{A}_{0}(\hat{y}) d \hat{y} \quad \text { for } k=2,3 .
$$

Here $\hat{A}_{0}(\hat{y})$ remains arbitrary, so the integral shown also remains arbitrary, and hence (5.9b) acts as a single constraint of the form $\lambda_{3} \mu_{2}=\lambda_{2} \mu_{3}$ on $\mu_{2}, \mu_{3}$. The solution is thereby completed.

The initial-value linearized problem in which appropriate conditions are imposed at time $t=0$ returns us to the system (5.3a)-(5.3i) of course. The solution then can be expressed in terms of the eigenmodes described in the previous paragraph, with a velocity potential $\phi$ satisfying $\tilde{u}=\partial \phi / \partial x$ again, (5.6a)-(5.6e) with $Q$ replaced by $\partial / \partial t$, and $\phi$ being cubic in $x$ as in (5.6f) essentially. In particular,

$$
\phi=\sum_{r=1}^{3} \sum_{k=0}^{3}\left(x-\frac{1}{2}\right)^{k} \hat{A}_{k}^{(r)}(\hat{y}) \exp \left(Q^{(r)} t\right),
$$

where the three roots of the cubic equation (5.8) are written as $Q=Q^{(r)}$ for $r=1,2,3$. The functions $\hat{A}_{0}^{(r)}$ are determined by the initial conditions at $t=0$, while the other three functions $\hat{A}_{k}^{(r)}$ for $k=1,2,3$ then follow from the reasoning concerned with (5.9a) and (5.9b). Thus the linear initial-value problem combined with the equations and conditions in (5.3a) and (5.3i) appears to be well posed, and it leads in general to an exponential growth of the perturbations, inferred from the positive root of (5.8), at large times. This finding applies for any $O(1)$ values of the scaled mass and moment of inertia $M, I$ since the continuum theory is independent of those parameters, and the same point applies to the nonlinear system (5.1a)-(5.1i) and the stability result (5.8).

\section{§6. Further comments.}

6.1. The continuum limit. An immediate point to make is that the continuumlimit behaviour suggested in the previous section goes through similarly for other effective shapes $R$ of grain or thin body just as well as for the straight shapes examined in detail above. In particular, it would be interesting to study 
the nonlinear form (5.1a)-(5.1i) numerically and the possible clashes within the system for any grain shape function $R$, where this function acts also as a slope or a density distribution within the continuum equations obtained. We note that lateral pressure gradients are non-zero in effect in the continuum-limit case, despite the presence of thin gaps and thin bodies throughout. A similar point about effective lateral pressure gradients arises in the study of multiple branching flows in [46]. It is noteworthy also in the present setting of nonlinear interactions between nearly parallel grains and the surrounding fluid that the results for relatively few grains (in the range 11-21, say) as described in $\$ 3$ shows signs of independence of grain number emerging, thus implying support for the continuum-limit model of $\S 5$.

A closely similar continuum-limit structure holds for non-straight leading edges and trailing edges and also for successive patches of grains.

The continuum limit as described in the previous section is independent of the mass and moment of inertia of the grains; here as well as in the events concerned with clashing summarized below the fluid-dynamic pressures have to adjust to produce zero net force and torque on the grain(s) effectively. This feature of the continuum theory assumes the scalings of mass and moment presented originally in $\$ 2$ are little altered as the number of grains increases. The feature can be enhanced simply, however, to allow for more substantial grain mass and moment. It is of further importance that the limit theory in $\S 5$ holds also for the lubrication regime which has viscous domination and for the intermediate interacting unsteady boundary layer regime which has a viscous-inviscid balance as well as for the inviscidly dominated regime at the heart of this paper.

6.2. Clashes. Concerning clashes or contacts between grains, most cases examined in the computational study in $\$ 3$ yield a leading-edge clash, that is, a clash focused at the leading edge of the configuration between just two grains or between a grain and a wall. Flat-on clashes where contact happens along the entire length of the grain(s) may also occur in principle and have been considered in several papers in settings quite different from the present one, while trailingedge clashes similarly seem likely to be possible in principle, but these have not been observed in any full-scale computational solutions so far and they are not addressed here. The analysis in $\S 4$ points to a rather sensitive dependence on time (see (4.7a), (4.7b)) as the finite-time clash appears and this type of dependence seems to be borne out by the comparisons with the computational behaviour of $\S 3$. We should observe again here that the analysis predicts a first possible term governing the clashing process and there may be circumstances in which the coefficient of that term is identically zero in practice. In similar vein, the main result for two grains clashing at the leading edge is exactly the same as for one grain hitting a wall as in (4.7a) and (4.7b). Following on also from $\S 4$ is the intriguing question of whether the grain-fluid interaction during a clash becomes mainly a local or a global phenomenon. The answer is "local" just by a fraction according to the comparison between the induced pressures in (4.6a) and (4.6b) that leads to the temporal response in (4.7a) and (4.7b). Moreover, the mass and moment of inertia of the grains typically play no part at leading 
order in the sense that the motion of the typical grain itself becomes a secondary influence, in a similar way to that of a grain near a side wall.

It would be interesting to explore the effects of thickness and camber here, as well as non-aligned leading and trailing edges as in Figure 1(b). These extensions involve essentially the same fluid-grain interactions as above.

6.3. Instabilities. Apart from the continuum limit and the finite-time clashing process above, a highlight of the grain-fluid model is that an apparently novel instability is present. Here the exponential growth involved as found in $\$ 3.2$ for one grain and in $\$ 5$ for many grains in effect is for some cases relatively small, indicating perhaps that the ideal of perfectly parallel grain movement might persist for some while, for instance in the higher parts of a chute. Eventually, however, the growth does force the system into the nonlinear stage of $\$ 3.1$ and thence, we believe, typically into a clash. Very near a wall the instability is dominated by the local gap effects only, as if for a grain which has zero mass and zero moment of inertia as described at the start of $\$ 5$. It would be interesting to extend the instability analysis to deal with any number of grains but, of more importance probably, to admit three spatial dimensions.

6.4. Follow-on issues. Many further questions of practical and/or theoretical concern arise. These concern especially: phenomena associated with bouncing of the grains straight after a clash and even repeated clashes; three-dimensional effects as mentioned already; non-straight side wall shapes concerned with chute shape design; the question of whether a grain or an array of grains can get stuck in a bend, modelling rice grains in a chute or bodies entering an engine intake; top-heavy or bottom-heavy grains where the centre of mass is adjusted; stability behaviour for many different configurations (e.g. varying parameters in $\$ 3.2$; see below); other shapes of grain, particularly with regard to the leading edge at a clash; applying the present findings on interactions at comparatively high flow rates.

Other issues surround the intermediate regime mentioned earlier (e.g. what becomes then of the self-supporting interplay found in \$3) as well as the extremes of relatively small or large grain mass or moment of inertia. One particular issue is the extension to wider chutes, or side walls spaced further apart, and even to grain-fluid interactions without side walls. This extension, which amounts to allowing the longitudinal and lateral length scales to be more wide-ranging, appears to be perfectly feasible by means of the continuum limit for example, since the main assumption in the continuum formulation is local, that the representative gap width and body width are small, and the effective global width between the side walls acts only as a passive factor. The side walls can also be completely removed in the discrete case on the assumption that the variations in gap pressure greatly exceed the ambient variations, as in the clashing phenomena.

Connecting back further with the original applications, however remotely, we observe that the geometric factors (e.g. the ratio $\beta$ ) involved in determining the scaled mass $M$ and moment of inertia $I$ can affect those values considerably. 
They could possibly be manipulated to produce by design an $M$ value below one-third, to reduce instability as in $\S 4$. For the food-sorting example, however, 150 grains on a chute of width $30 \mathrm{~cm}$ produce a representative $\beta$ of about 0.4. Coupled with a representative mass $M^{*}$ for a rice grain of $0.025 \mathrm{~g}$ and density $\rho^{*}$ for air of $0.0012 \mathrm{~g} \mathrm{~cm}^{-3}$, this gives $M$ equal to about 30; even allowing for appreciable three-dimensional factors seems unlikely to reduce the value below $1 / 3$. On the other hand the effects of moving the centre of mass, for instance, as well as the role of grain thickness in the grain-fluid interactions need further study here. There is also the important extension to three dimensions to be considered as stated previously, even if the broad findings of clashes, instability and continuum effects remain unaltered. Concerning the nominally infinite velocity of fluid at the leading edge $(\S 4)$ at a clashing the present analysis will actually break down just before solid-solid contact due to thickness, viscous, Knudsen or surface-roughness effects and presumably lead to a finite collision velocity in particular. The subsequent phenomena during and after collision also merit further investigation, although qualitatively we might expect the process of fluid-solid interaction in $\S \S 2-5$ to repeat itself after a bouncing between bodies or between a body and a wall.

Acknowledgements. Thanks are due to EPSRC for support of ASE and to present and former staff at QinetiQ (Roger Gent and colleagues) and Sortex Ltd (Gabriel Hamid, Mark Honeywood and colleagues). We are also grateful to Icing Group members and Industrial Mathematics KTN members for their encouragement and interest.

\section{References}

1. H. Ahn, C. E. Brennen and R. H. Sabersky, Analysis of the fully developed chute flow of granular materials. J. Appl. Mech. $\mathbf{5 9}$ (1992), 109.

2. A. M. Ardekani, R. H. Rangel and D. D. Joseph, Motion of a sphere normal to a wall in a secondorder fluid. J. Fluid Mech. 587 (2007), 163-172.

3. M. Andrew, M. David, G. deVeber and L. A. Brooker, Arterial thromboembolic complications in paediatric patients. Thromb. Haemost. 78(1) (1997), 715-725.

4. A. Argentina and L. Mahadevan, Fluid-flow-induced flutter of a flag. Proc. Natl. Acad. Sci. 102(6) (2005), 1829-1834.

5. P. S. Babyn, H. K. Gahunia and P. Massicotte, Pulmonary thromboembolism in children. Pediatr. Radiol. 35(3) (2005), 258-274.

6. W. F. Baker, Diagnosis of deep venous thrombosis and pulmonary embolism. Med. Clin. North Am. 82(3) (1998), 459-476.

7. A. Belmonte, H. Eisenberg and E. Moses, From flutter to tumble: inertial drag and Froude similarity in falling paper. Phys. Rev. Lett. 81 (1998), 345.

8. R. G. A. Bowles and Smith, Lifting multi-blade flows with interaction. J. Fluid Mech. 415 (2000), 203-226.

9. R. I. Bowles, S. C. R. Dennis, R. Purvis and F. T. Smith, Multi-branching flows from one mother tube to many daughters or to a network. Philos. Trans. R. Soc. A 363 (2005), 1045-1055.

10. R. I. Bowles, N. C. Ovenden and F. T. Smith, Multi-branching 3D flow with substantial changes in vessel shapes. J. Fluid Mech. 614 (2008), 329-354.

11. C. S. Campbell, Rapid granular flows. Annu. Rev. Fluid Mech. 22 (1990), 57.

12. J. K. Comer, C. Kleinstreuer and C. S. Kim, Flow structures and particle deposition patterns in double-bifurcation airway models. Part 2. Aerosol transport and deposition. J. Fluid Mech. $\mathbf{4 3 5}$ (2001), 55-80.

13. I. Eames, J. C. R. Hunt and S. E. Belcher, Inviscid mean flow through and around groups of bodies. J. Fluid Mech. 515 (2004), 371. 
14. H. Ehrentraut and A. Chrzanowska, Induced anisotropy in rapid flows of nonspherical granular materials. In Dynamic Response of Granular and Porous Materials under Large and Catastrophic Deformations, Vol. 11 (eds K. Hutter and N. Kirchner), Springer (Berlin, 2003), 343-364.

15. A. S. Ellis, Modelling chute delivery of grains in a food-sorting process. PhD Thesis, University of London, 2007.

16. A. S. Ellis and F. T. Smith, A continuum model for a chute flow of grains. SIAM J. Appl. Math. 69(2) (2008), 305-329.

17. A. F. Fortes, D. D. Joseph and T. S. Lundgren, Nonlinear mechanics of fluidization of beds of spherical particles. J. Fluid Mech. 177 (1987), 467-483.

18. D. P. Gaver, D. Halpern and O. E. Jensen, Surfactant and airway liquid flows. In Lung Surfactant and Disorder: Lung Biology in Health and Disease (ed. K. Nag), Taylor and Francis (Boca Raton, 2005), 201.

19. J. M. N. T. Gray and K. Hutter, Pattern formation in granular media. Contin. Mech. Thermodyn. 9 (1997), 341.

20. E. Guazzelli, Sedimentation of small particles: how can such a simple problem be so difficult? C. $R$. Mécanique 334 (2006), 539-544.

21. S. R. Hodges, O. E. Jensen and J. M. Rallison, The motion of a viscous drop through a cylindrical tube. J. Fluid Mech. 501 (2004), 279-301.

22. H. E. Huppert, Quantitative modelling of granular suspension flows. Philos. Trans. R. Soc. Lond. A 356 (1998), 2471.

23. P. Y. Huang, J. Feng and D. D. Joseph, The turning couples on an elliptic particle settling in a vertical channel. J. Fluid Mech. 271 (1994), 1-16.

24. Y. Iguchi and K. Kimura, A case of brain embolism during catheter embolisation of head arteriovenous malformation. What is the mechanism of stroke? J. Neurol. Neurosurg. Psychiatry 78 (2007), 81.

25. J. T. Jenkins and S. B. Savage, A theory for the rapid flow of identical, smooth, nearly elastic, spherical particles. J. Fluid Mech. 130 (1983), 187.

26. J. T. Jenkins, Boundary conditions for rapid granular flows: flat, frictional walls. J. Appl. Mech. 59 (1992), 120.

27. L.-B. Jia, F. Li, X.-Z. Yin and X.-Y. Yin, Coupling modes between two flapping filaments. J. Appl. Mech. 581 (2007), 199-220.

28. M. A. Jones and F. T. Smith, Fluid motion for car undertrays in ground effect. J. Engrg. Math. 45 (2003), 309-334.

29. L. P. Kadanoff, Built upon sand: theoretical ideas inspired by granular flows. Rev. Modern Phys. 71 (1999), 435.

30. D. L. Koch and R. J. Hill, Inertial effects in suspension and porous-media flows. Annu. Rev. Fluid Mech. 33 (2001), 619.

31. A. A. Korobkin, Impact of two bodies one of which is covered by a thin layer of liquid. J. Fluid Mech. 300 (1995), 43-58.

32. A. A. Korobkin and M. Ohkusu, Impact of two circular plates one of which is floating on a thin layer of liquid. J. Engrg. Math. 50 (2004), 343-358.

33. M. Y. Louge, Computer simulations of rapid granular flows of spheres interacting with a flat frictional boundary. Phys. Fluids 6 (1994), 2253.

34. J. Magnaudet and I. Eames, Dynamics of high Re bubbles in inhomogeneous flows. Annu. Rev. Fluid Mech. 32 (2000), 659-708.

35. R. Monetti, A. Hurd and V. M. Kenkre, Simulations for dynamics of granular mixtures in a rotating drum. Granular Matter. 3 (2001), 113.

36. J. N. Newman, Analysis of small-aspect-ratio lifting surfaces in ground effect. J. Fluid. Mech. 117 (1982), 305-314.

37. N. C. Ovenden, F. T. Smith and G.-X. Wu, Effects of nonsymmetry in a branching flow network. J. Engrg. Math. 63 (2008), 213-239.

38. K. Pancholi, E. Stride and M. Edirisinghe, Dynamics of bubble formation in highly viscous liquids. Langmuir 24 (2008), 4388-4393.

39. R. Purvis and F. T. Smith, Planar flow past two or more blades in ground effect. Q. J. Mech. Appl. Math. 57(1) (2004), 137-160. 
40. J. Rajchenbach, Granular flows. Adv. Phys. 49 (2000), 229.

41. J. A. Schonberg and E. J. Hinch, Inertial migration of a sphere in Poiseuille flow. J. Fluid Mech. 203 (1989), 517-524.

42. T. W. Secomb, R. Skalak, N. Özkaya and J. F. Gross, Flow of axisymmetric red blood cells in narrow capillaries. J. Fluid Mech. 163 (1986), 405-423.

43. F. T. Smith and S. N. Timoshin, Blade-wake interactions and rotary boundary layers. Proc. R. Soc. A 452 (1996a), 1301-1329.

44. F. T. Smith and S. N. Timoshin, Planar flows past thin multi-blade configurations. J. Fluid Mech. 324 (1996b), 355-377.

45. F. T. Smith and M. A. Jones, One-to-few and one-to-many branching tube flows. J. Fluid Mech. 423 (2000), 1-31.

46. F. T. Smith and M. A. Jones, AVM modelling by multi-branching tube flow: large flow rates and dual solutions. Math. Medicine Biology 20 (2003), 183-204.

47. F. T. Smith, L. Li and G.-X. Wu, Air cushioning with a lubrication/inviscid balance. J. Fluid Mech. 482 (2003), 291-318.

48. F. T. Smith, N. C. Ovenden and R. Purvis, Industrial and biomedical applications. Solid Mech. Appl. 129(5) (2006), 291-300.

49. H. A. Stone, A. D. Stroock and A. Ajdari, Engineering flows in small devices: micro-fluidics toward a lab-on-a-chip. Annu. Rev. Fluid Mech. 36 (2004), 381-411.

50. E. O. Tuck, A nonlinear unsteady one-dimensional theory for wings in extreme ground effect. J. Fluid Mech. 98 (1980), 33-47.

51. E. O. Tuck and T. M. Bentwich, Sliding sheets: lubrication with comparable viscous and inertia forces. J. Fluid Mech. 41 (1983), 769-792.

52. E. Villermaux and C. Clanet, Life of a flapping liquid sheet. J. Fluid Mech. 462 (2002), 341-363.

53. A. H. White, Mathematical modelling of the embolisation process in the treatment of arteriovenous malformations. PhD Thesis, University of London, 2008.

54. B. Willetts, Aeolian and fluvial grain transport. Philos. Trans. R. Soc. Lond. A 356 (1998), 2471.

55. H. J. Wilson and R. H. Davis, The viscosity of a dilute suspension of rough spheres. J. Fluid Mech. 421 (2000), 339.

56. H. J. Wilson and R. H. Davis, Shear stress of a monolayer of rough spheres. J. Fluid Mech. 452 (2002), 425.

57. J. Xu, M. R. Maxey and G. E. Karniadakis, Numerical simulation of turbulent drag reduction using micro-bubbles. J. Fluid Mech. 468 (2002), 271-281.

58. B. H. Yang, J. Wang, D. D. Joseph, H. H. Hu, T. W. Pan and R. Glowinski, Migration of a sphere in a tube flow. J. Fluid Mech. $\mathbf{5 4 0}$ (2005), 109-131.

59. C.-S. Yih, Fluid mechanics of colliding plates. Phys. Fluids 17 (1974), 1936-1940.

Frank T. Smith,

Department of Mathematics,

UCL,

Gower Street, London WC1E 6BT,

U.K.

E-mail: frank@math.ucl.ac.uk
Andrew S. Ellis,

Department of Mathematics,

UCL,

Gower Street, London WC1E 6BT,

U.K. 